\title{
FORGOTTEN CARPOLOGICAL COLLECTION OF PROFESSOR IVO HORVAT DISCOVERED AND DIGITIZED
}

\author{
Sara Essert ${ }^{1}$, Vedran Šmota $^{2 *}$, Ivana God ${ }^{3}$, Nora Mas ${ }^{4}$, \\ Daniel Šroljarić ${ }^{5} \&$ Maja Popović 5
}

${ }^{1}$ Division of Botany, Department of Biology, Faculty of Science, University of Zagreb, Marulićev trg 20/II, HR-10000 Zagreb, Croatia

${ }^{2} \mathrm{ZA} \&$ ZAHO herbarium collections, Division of Botany, Department of Biology, Faculty of Science, University of Zagreb, Marulićev trg 20/II, HR-10000 Zagreb, Croatia

${ }^{3}$ Ivan Kukuljević Sakcinski Elementary School, Akademika Ladislava Šabana 17, HR-42240 Ivanec, Croatia

${ }^{4}$ Department of Animal Nutrition and Dietetics, Faculty of Veterinary Medicine, University of Zagreb, Heinzelova 55, HR-10000 Zagreb, Croatia

${ }^{5}$ Department of Veterinary Biology, Faculty of Veterinary Medicine, University of Zagreb, Heinzelova 55, HR-10000 Zagreb, Croatia

Essert, S., Šegota, V., God, I., Mas, N., Špoljarić, D. \& Popović, M.: Forgotten carpological collection of Professor Ivo Horvat discovered and digitized. Nat. Croat. Vol. 30, No. 1., 269-287, 2021, Zagreb.

The carpological collection of Professor Ivo Horvat, a famous Croatian botanist of $20^{\text {th }}$ century, was saved from oblivion, after its unexpected discovery at the Faculty of Veterinary Medicine in Zagreb in 2019. As many as 515 diaspores (fruits and seeds) of 486 vascular plant taxa were systematised and digitised and the nomenclature was updated. A comprehensive comparison of Horvat's carpological and herbarium collection $(\mathrm{ZAHO})$ revealed a large amount of overlap. A large photo-catalogue was created and will be publicly accessible through the Flora Croatica Database.

Key words: diaspores, fruits, herbarium, photo-catalogue, seeds, vascular plants

Essert, S., Šegota, V., God, I., Mas, N., Špoljarić, D. \& Popović, M.: Pronađena i digitalizirana zaboravljena karpološka zbirka profesora Ive Horvata. Nat. Croat. Vol. 30, No. 1., 269-287, 2021, Zagreb.

Karpološka zbirka profesora Ive Horvata, velikog hrvatskog botaničara 20. stoljeća, sačuvana je od zaborava nakon neočekivanog pronalaska na Veterinarskom fakultetu u Zagrebu tijekom 2019. godine. Ukupno 515 dijaspora (plodova i sjemenki) koje pripadaju 486 svojti vaskularne flore je sistematizirano i digitalizirano, a nomenklatura je ažurirana. Sveobuhvatna usporedba Horvatove karpološke i herbarijske zbirke (ZAHO) rezultirala je velikom količinom preklapanja. Formiran je veliki fotokatalog koji će biti javno dostupan u bazi podataka Flora Croatica.

Ključne riječi: dijaspore, plodovi, herbarij, fotokatalog, sjemenke, vaskularna flora

\section{INTRODUCTION}

Professor Ivo Horvat (1897-1963) was one of the most prominent Croatian botanists and one of the most active explorers of the vegetation of the former Yugoslavia and the Balkan Peninsula (Bertović, 1963). During his 40 years of scientific work as a

* corresponding author: vedran.segota@biol.pmf.hr 
pioneer in vegetation studies based on the principles of the Braun-Blanquet phytosociological school, he became one of the leading phytocoenologists at that time in Europe. He started to work as a professor of botany at the former Institute of Botany at the Faculty of Science of the University of Zagreb, but the majority of his professional life was spent working at the former Department of Botany of the Faculty of Veterinary Medicine of the same university (Horvatić, 1963). He published more than 80 scientific and 90 professional papers (BERTOVIĆ, 1963). During his numerous field trips, he collected a huge amount of plant material, systemized after his death in the Herbarium of Ivo and Marija Horvat (ZAHO) (TheIrs, 2020), which was finally, after many relocations, housed in the Botanical Garden of the Faculty of Science in Zagreb in 1998. The collection contains 71,611 herbarium sheets collected mostly in Croatia and South-East Europe (Horvat \& Plazibat, 2007).

Carpological collections, specialized herbarium collections containing only diaspores (fruits and seeds) of vascular plants, are mostly kept within botanical gardens and are regularly used in education as well as in scientific studies dealing with seed germination ecology. The best-known seed bank, the Svalbard Global Seed Vault on the Norwegian island of Spitsbergen, keeps as many as 860.000 seeds of various vascular plants (wild and cultivated crops, rare and endangered taxa etc.). Currently, there are around 1,400 seed banks in 100 countries, preserving global plant genetic diversity (BOLF, 2018). In addition, carpological collections are regularly used as comparative compendia in archaeobotanical studies of plant macrofossils (CAPPERS \& Neef, 2012; Pearsall, 2000).

\section{MATERIALS AND METHODS}

The carpological collection of Professor Ivo Horvat was surveyed and analysed at the Division of Botany of the Faculty of Science during 2019. The nomenclature of the taxa was validated and updated based on the Flora Croatica Database (Nikolić, 2019) and The Plant List (2019). For each collected taxon, affiliation to plant family, endemic, threatened and strictly protected flora were inspected (ANONYMOUs, 2016; Nikolić \& Topić, 2005; Nikolić, 2019). An effort was made to compare the taxa from the studied carpological collection with the herbarium specimens from the Herbarium of Ivo and Marija Horvat (ZAHO). All herbarium sheets potentially connected to the carpological collection were digitised following the standard procedure (ŠEGOTA et al., 2017). Finally, the whole carpological collection was photographed - each glass container with numerous diaspores with a Huawei p20 smartphone, and several isolated diaspores of each taxon by USB Dino-Lite Pro HR, AM7000/AD7000 series (5 megapixels) digital microscope, resulting in a catalogue of 486 seeds and fruits.

\section{RESULTS AND DISCUSSION}

This large carpological collection was found by mere chance in 2019 in the cellar of the Faculty of Veterinary Medicine. It was hypothesized that it belonged to Professor Ivo Horvat, a botanist who had worked there from 1947 to 1963. However, no written evidence of the existence of this collection was identified, either in his bibliography or in several posthumous biographies (e.g. Bertović, 1963; Horvatić, 1963). Moreover, not a single mention of this collection was found among the professor's scientific or private documents and handbooks, while his relatives have no information about his work with the carpological collection. We discovered that in the 1960s the collection 


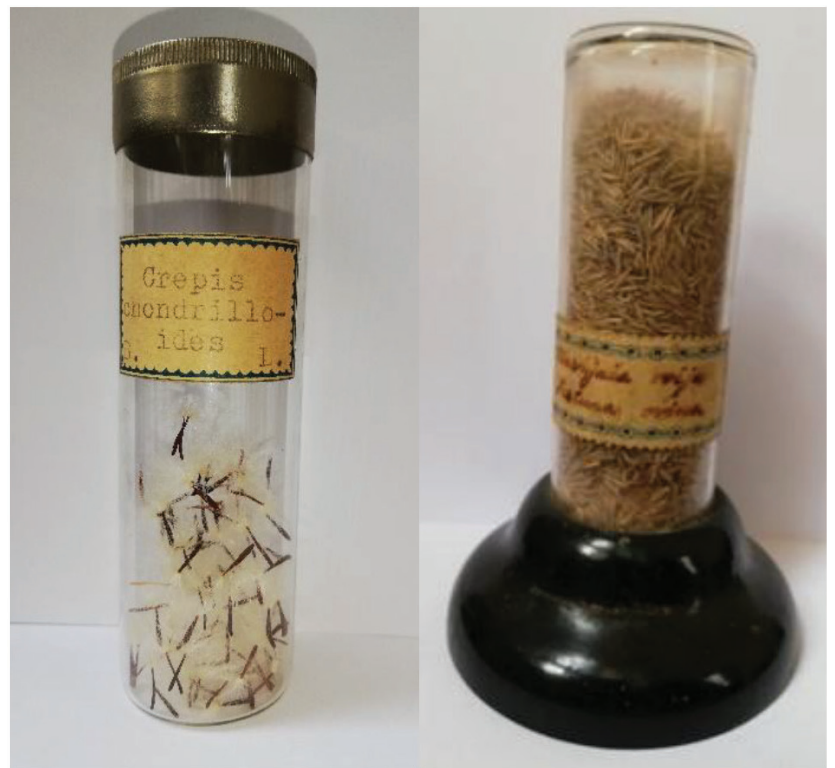

Fig. 1. Exemplars of the glass containers with metal cap (left) and on wooden stand (right) from Horvat's carpological collection.

was moved to the cellar of the Faculty of Veterinary Medicine where it was managed for some time by Dr Željko Horvat and subsequently faded into oblivion. However, it is highly probable that this collection was compiled by Professor Horvat while collecting numerous plants for his scientific herbarium, as well as for teaching material for botany lectures.

We summarized the collection of as many as 515 tube-like glass containers with metal caps and the labels with handwritten or typed Latin name of the taxon (Fig. 1). Five containers were different, with a wooden stand, used probably as exemplars during lectures. No other basic metadata such as locality, habitat, collecting date or collector were found on the labels, with the exception of just three labels on which localities were noted (Prunus spinosa L. - Krk \& Zagreb, Abies alba Mill. - G. Kotar). Repetitions were found only in 12 taxa, one (Medicago lupulina L.) being collected in three, and 11 (Biscutella laevigata L., Festuca pratensis Huds., Hypochaeris maculata L., Laurus nobilis L., Lycopus europaeus L., Medicago sativa L., Pinus halepensis Mill., Trifolium incarnatum L., Trifolium pratense L, Prunus spinosa L., Vicia sativa L.) in two containers. After the analysis, the collection was returned and deposited within the Department of Veterinary Biology of the Faculty of Veterinary medicine in Zagreb (Fig. 2).

The comprehensive inventory and nomenclature update revealed as many as 486 vascular plant taxa (Appendix 1). A nomenclature validation was necessary for $19.75 \%$ of inspected taxa names. As many as $95.28 \%$ of the taxa are members of the Croatian flora (Nikolić, 2019) indicating that the majority of the collection was most likely collected in Croatia or neighbouring countries, as was also the case with Horvat's herbarium collection (Horvat \& Plazibat, 2007). The collected specimens belong to 102 plant families, with Asteraceae and Fabaceae (10.88 \% each) and Poaceae $(7.59 \%)$ being the most abundant (Tab. 1). 


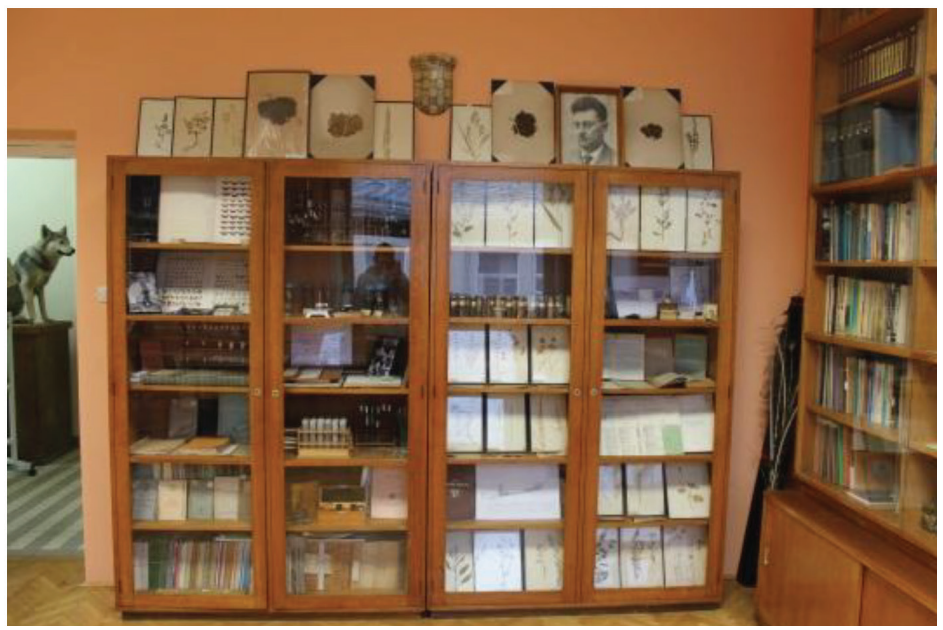

Fig. 2. The current location of Horvat's carpological collection at the Department of Veterinary Biology of the Faculty of Veterinary Medicine in Zagreb.

Tab. 1. Families with the highest number of taxa within Horvat's carpological collection.

\begin{tabular}{|c|c|c|}
\hline Family & No. of taxa & \% of total flora \\
\hline Asteraceae & 53 & 10.88 \\
\hline Fabaceae & 53 & 10.88 \\
\hline Poaceae & 37 & 7.59 \\
\hline Brassicaceae & 23 & 4.70 \\
\hline Apiaceae & 22 & 4.52 \\
\hline Lamiaceae & 22 & 4.52 \\
\hline Cichoriaceae & 21 & 4.31 \\
\hline Rosaceae & 19 & 3.90 \\
\hline Caryophyllaceae & 10 & 2.04 \\
\hline Ranunculaceae & 10 & 2.04 \\
\hline Scrophulariaceae & 10 & 2.04 \\
\hline Solanaceae & 9 & 1.85 \\
\hline Asparagaceae & 8 & 1.64 \\
\hline Euphorbiaceae & 7 & 1.43 \\
\hline Malvaceae & 7 & 1.43 \\
\hline Polygonaceae & 7 & 1.43 \\
\hline Rutaceae & 7 & 1.43 \\
\hline Caprifoliaceae & 6 & 1.23 \\
\hline Pinaceae & 6 & 1.23 \\
\hline other families $(91)$ & 150 & 30,91 \\
\hline & & \\
\hline & & \\
\hline
\end{tabular}

Six endemic taxa (Tanacetum cinerariifolium, Edraianthus tenuifolius, Iris illyrica, Leucanthemum atratum ssp. platylepis, Peltaria alliacea and Chouardia litardierei) and 28 Redlisted, three of which critically endangered (Digitalis lanata, Ligularia sibirica and Plantago indica) were found. According to the latest legislation, 19 taxa from the collection 
are currently strictly protected in Croatia (Achillea ptarmica, Alopecurus rendlei, Arnica montana, Tanacetum cinerariifolium, Digitalis lanata, Edraianthus tenuifolius, Glaucium flavum, Hibiscus trionum, Iris pseudacorus, Iris sibirica, Ligularia sibirica, Lilium martagon, Paeonia mascula, Peltaria alliacea, Plantago subulata, Plantago indica, Taxus baccata and Trapa natans).

Comparison between Horvat's carpological and herbarium (ZAHO) collections revealed overlapping in 334 (68.58 \%) taxa (Fig. 3, Fig. 4). As many as $78.44 \%$ of those taxa have herbarium specimens with mature fruits and seeds. Among them, only $20.10 \%$ of taxa from carpological collection could be related to herbarium specimens collected at solely one locality.
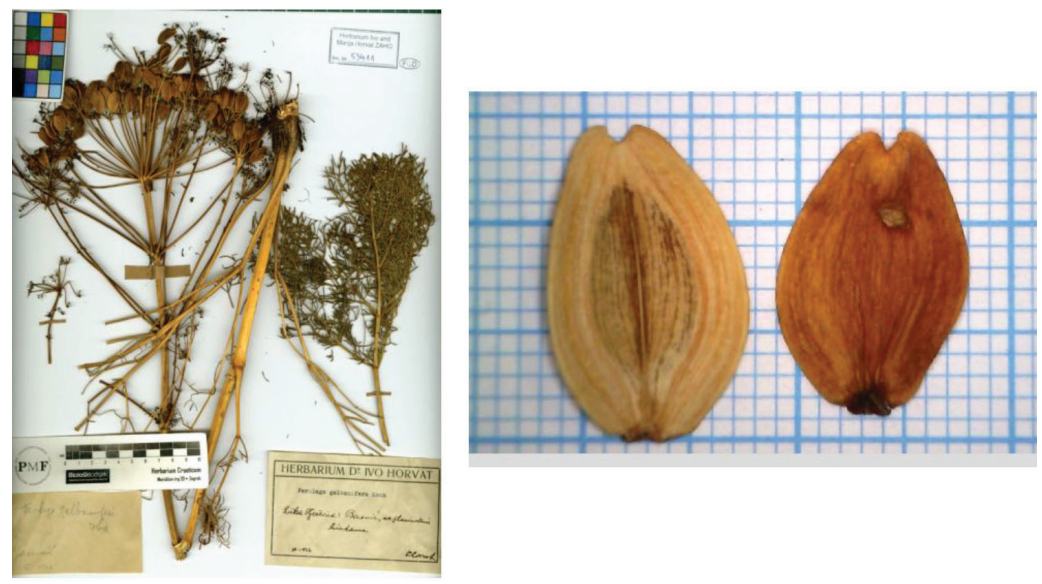

Fig. 3. Specimens of Ferulago campestris (Besser) Grecescu from Horvat's herbarium (ZAHO) and its fruits from the carpological collection.
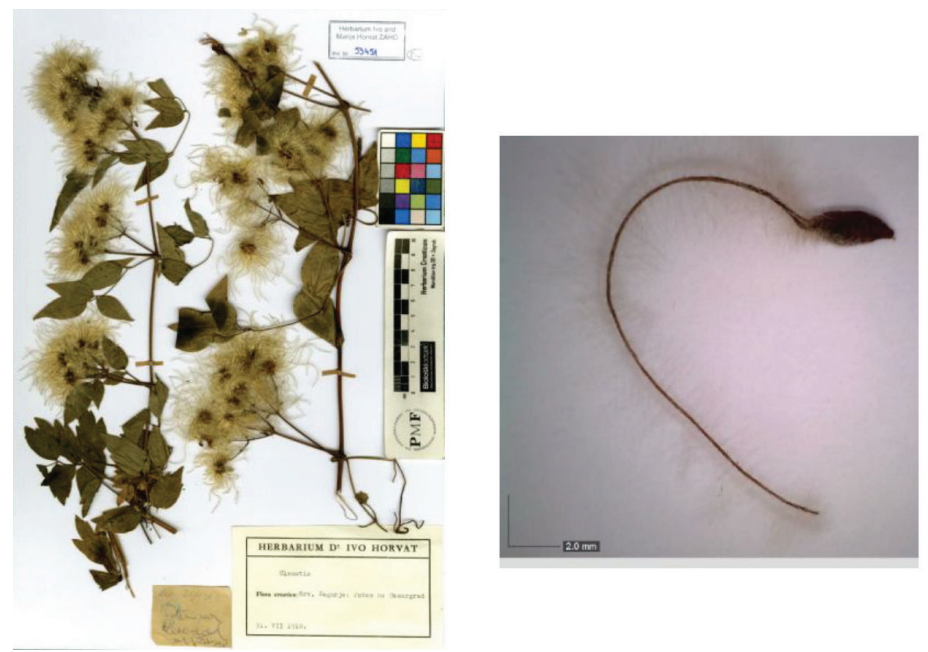

Fig. 4. Specimens of Clematis vitalba L. from Horvat's herbarium (ZAHO) and its fruits from carpological collection. 


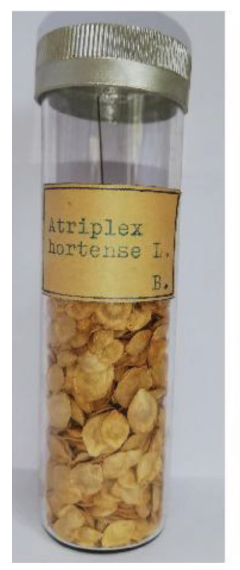

Atriplex hortensis L.
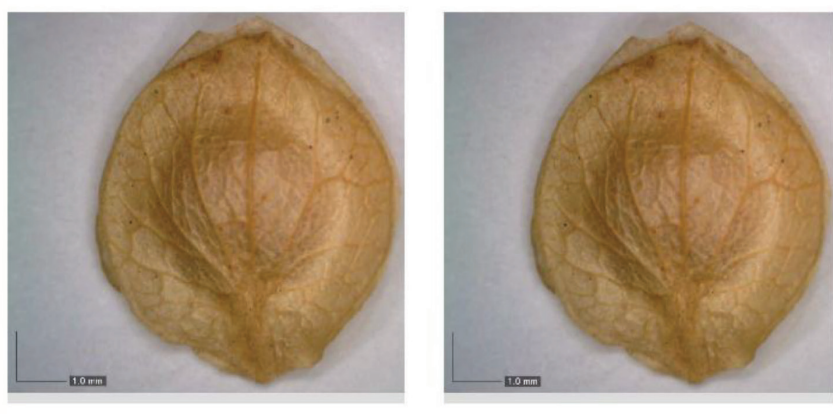

Fig. 5. Illustration of one species (Atriplex hortensis L.) within the photo-catalogue of the Horvat carpological collection.

The remaining $21.56 \%$ of inspected taxa herbarium specimens lacked diaspores, or fruits and seeds were collected immature. Based on this data, we could only assume that Horvat collected simultaneously plants for herbarium and their diaspores for carpological collection. However, about half of the taxa (51.20\%) specimens with mature fruits were collected from two or more localities. It is possible that the collection was also partially created by material exchange with other local and foreign botanists, that the material was collected simultaneously by several collectors that cooperated with Professor Horvat, or that part of the collection was obtained from other foreign collections for lecture purposes. We have no evidence for any of these scenarios. Since there are no written documents indicating that this collection was actually used in lecture processes at the Faculty of Veterinary Medicine in Zagreb, further study should include interviews with Horvat's students who possibly have memories of this collection. Unfortunately, since this collection was probably not used after the 1960s, locating witnesses would be rather challenging.

The photo-catalogue of the collection contains as many as 1186 pictures and represents the first comprehensive digitised photo-catalogue of diaspores in Croatia (Fig. 5). Pictures are currently stored in the Archaeobotanical Laboratory (Division of Botany, Faculty of Science) and will soon be published and made publicly available in the Flora Croatica Database. Since this database contains mostly pictures of plant habitus, leaves and flowers, the addition of pictures of fruits and seeds will certainly be a valuable contribution and helpful tool for all users during plant identifications. Finally, several diaspores of each taxon were extracted from Horvat's collection and included in the carpological collection of the Archaeobotanical Laboratory (Division of Botany, Faculty of Science).

\section{ACKNOWLEDGMENTS}

This research is a part of the collaborative project of the Croatian Herbarium (ZA) at the Faculty of Science and the Department of Veterinary Biology at the Faculty of Veterinary Medicine on the evaluation of the legacy of Professor Ivo Horvat. 


\section{REFERENCES}

Anonymous, 2016: Pravilnik o izmjenama i dopunama pravilnika o strogo zaštićenim vrstama. Narodne novine, $73 / 2016$.

Bertović, S., 1963: Prof. dr. Ivo Horvat - životopis, znanstveni rad i ostavština. Biološki glasnik 16(3-4), 13-29.

Bolf, N., 2018: Svjetska banka sjemena na Svalbardu. Kemija u industriji 67(9-10), 429-430.

Cappers, R. T. J. \& Neef, R, 2012: Handbook of Plant Paleoecology. Barkhuis Groningen University Library. Groningen.

Horvat, M. \& Plazibat, M., 2007: Prikaz Horvatova herbarija (ZAHO) u Zagrebu. Natura Croatica 16(4), 267-408.

Horvatić, S., 1963: Ivo Horvat - In memoriam. Acta Botanica Croatica, 22(1), 13-25.

Nikolić, T. \& Topić, J., 2005: Crvena knjiga vaskularne flore Hrvatske. Ministartsvo kulture, Državni zavod za zaštitu prirode, Zagreb.

Nikolić, T. (ed.) (2019): Flora Croatica Database. On-Line (http://hirc.botanic.hr/fcd). Division of Botany, Faculty of Science, University of Zagreb, accessed on 1 July 2019.

Pearsall, D., 2000: Paleoethnobotany, a handbook of procedures. Academic press, San Diego.

ReŠEtnik, I. \& ŠEGOTA, V., 2020: Virtual herbarium ZA \& ZAHO. On-line (http:/ /herbariumcroaticum.biol.pmf.hr). Division of Botany, Faculty of Science, University of Zagreb, accessed on 1 July 2019.

Šegota, V., Buzjak, S., Vilović, T., Sedlar, Z., Rešetnik, I. \& Bogdanović, S., 2017: Curators in action: intricate genus Fritillaria L. (Liliaceae) from ZA, ZAHO, CNHM and ZAGR revised and digitized. Glasnik Hrvatskog botaničkog društva, 5(2), 4-14.

The Plant List, 2019: Version 1.1. On-line (http:/ / www.theplantlist.org), accessed on 1 July 2019.

Thiers, B., 2020: Index Herbariorum. On-Line (http://sweetgum.nybg.org/ih/) A global directory of public herbaria and associated staff. New York Botanical Garden's Virtual Herbarium, accesses on 1 January 2020.

\section{APPENDIX 1.}

List of taxa found in the carpological collection of Professor Ivo Horvat (HR - present in Croatian flora, ZAHO - present in Herbarium of Ivo and Marija Horvat)

\begin{tabular}{|c|c|c|c|c|c|}
\hline No. & original name on label & valid name & family & $\mathrm{HR}$ & ZAHO \\
\hline 1 & Abies alba Mill. & Abies alba Mill. & Pinaceae & $x$ & \\
\hline 2 & Abutilon theophrasti & Abutilon theophrasti Medik. & Malvaceae & $x$ & \\
\hline 3 & Acer monspessulanum L. & Acer monspessulanum L. & Aceraceae & $x$ & $x$ \\
\hline 4 & Acer negundo & Acer negundo L. & Aceraceae & $x$ & $x$ \\
\hline 5 & Acer pseudoplatanus L. & Acer pseudoplatanus L. & Aceraceae & $x$ & $x$ \\
\hline 6 & Achillea millefolium $\mathrm{L}$. & Achillea millefolium $\mathrm{L}$. & Asteraceae & $x$ & $x$ \\
\hline 7 & Achillea ptarmica L. & Achillea ptarmica L. & Asteraceae & $x$ & \\
\hline 8 & $\begin{array}{l}\text { Adenostyles alpina } \mathrm{Bl} \text {. et } \\
\text { Fingr. }\end{array}$ & $\begin{array}{l}\text { Adenostyles alpina (L.) Bluff et } \\
\text { Fingerh }\end{array}$ & Asteraceae & $x$ & \\
\hline 9 & Agrimonia pilosa Ledeb. & Agrimonia pilosa Ledeb. & Rosaceae & & \\
\hline 10 & Agrostemma githago L. & Agrostemma githago L. & Caryophyllaceae & $x$ & \\
\hline 11 & Agrostis alba L. & Agrostis gigantea Roth & Poaceae & $x$ & $x$ \\
\hline 12 & Ailanthus glandulosa & $\begin{array}{l}\text { Ailanthus altissima (Mill.) } \\
\text { Swingle }\end{array}$ & Simaroubaceae & $x$ & $\mathrm{x}$ \\
\hline 13 & Alisma plantago aquatica L. & Alisma plantago-aquatica L. & Alismataceae & $x$ & $x$ \\
\hline
\end{tabular}




\begin{tabular}{|c|c|c|c|c|c|}
\hline No. & original name on label & valid name & family & HR & $\mathrm{ZAHO}$ \\
\hline 14 & Alliaria officinalis Andr. & $\begin{array}{l}\text { Alliaria petiolata (M. Bieb.) } \\
\text { Cavara et Grande }\end{array}$ & Brassicaceae & $\mathrm{x}$ & $\mathrm{x}$ \\
\hline 15 & Allium porrum $\mathrm{L}$. & Allium porrum L. & Amaryllidaceae & $x$ & \\
\hline 16 & Alopecurus pratense L. & Alopecurus pratensis L. & Poaceae & $\mathrm{x}$ & \\
\hline 17 & Alopecurus utriculatus Pens. & Alopecurus rendlei Eig & Poaceae & $x$ & $x$ \\
\hline 18 & Altheae officinalis L. & Althaea officinalis L. & Malvaceae & $x$ & $\mathrm{x}$ \\
\hline 19 & $\begin{array}{l}\text { Alyssoides utriculatum (L.) } \\
\text { Med.J. }\end{array}$ & $\begin{array}{l}\text { Alyssoides utriculata (L.) } \\
\text { Medik. }\end{array}$ & Brassicaceae & $x$ & $\mathrm{x}$ \\
\hline 20 & Amaranthus silvestris Desf. & $\begin{array}{l}\text { Amaranthus graecizans ssp. } \\
\text { silvestris (Vill.) Brenan }\end{array}$ & Amaranthaceae & $\mathrm{x}$ & \\
\hline 21 & Amarantus caudatus L. & Amaranthus caudatus L. & Amaranthaceae & $\mathrm{x}$ & \\
\hline 22 & Amorpha fruticosa $\mathrm{L}$. & Amorpha fruticosa $\mathrm{L}$. & Fabaceae & $x$ & $x$ \\
\hline 23 & Anagallis arvensis L. & Anagallis arvensis L. & Primulaceae & $x$ & $\mathrm{x}$ \\
\hline 24 & Anemone nemorosa L. & Anemone nemorosa L. & Ranunculaceae & $x$ & $x$ \\
\hline 25 & Anethum graveolens L. & Anethum graveolens L. & Apiaceae & $\mathrm{x}$ & $\mathrm{x}$ \\
\hline 26 & Angelica archangelica L. & Angelica archangelica L. & Apiaceae & $x$ & \\
\hline 27 & Angelica silvestris L. & Angelica sylvestris L. & Apiaceae & $x$ & $\mathrm{x}$ \\
\hline 28 & Anoda hastata Cav. & Anoda cristata (L.) Schltdl. & Malvaceae & & \\
\hline 29 & Antennaria dioica L. & Antennaria dioica $\mathrm{L}$. & Asteraceae & $\mathrm{x}$ & $\mathrm{x}$ \\
\hline 30 & Anthericum ramosum $\mathrm{L}$. & Anthericum ramosum $\mathrm{L}$. & Asparagaceae & $x$ & $\mathrm{x}$ \\
\hline 31 & Anthoxanthum odoratum & Anthoxanthum odoratum L. & Poaceae & $x$ & $x$ \\
\hline 32 & Anthyllis jacquini Kern. & $\begin{array}{l}\text { Anthyllis montana ssp. jacquinii } \\
\text { (A. Kern.) Hayek }\end{array}$ & Fabaceae & $\mathrm{x}$ & $\mathrm{x}$ \\
\hline 33 & Anthyllis vulneraria L. & Anthyllis vulneraria L. & Fabaceae & $x$ & $\mathrm{x}$ \\
\hline 34 & Aposeris foetida $\mathrm{L}$. & Aposeris foetida (L.) Cass. ex Less. & Cichoriaceae & $x$ & $\mathrm{x}$ \\
\hline 35 & Arabis hirsuta & Arabis hirsuta (L.) Scop. & Brassicaceae & $x$ & \\
\hline 36 & Arabis turrita L. & Arabis turrita L. & Brassicaceae & $x$ & $\mathrm{x}$ \\
\hline 37 & Arachis hypogaea & Arachis hypogaea L. & Fabaceae & & \\
\hline 38 & Arenaria serphyllifolia L. & Arenaria serpyllifolia $\mathrm{L}$. & Caryophyllaceae & $\mathrm{x}$ & $\mathrm{x}$ \\
\hline 39 & Aristolochia clematitis L. & Aristolochia clematitis L. & Aristolochiaceae & $x$ & $x$ \\
\hline 40 & Armeria canescens Host. & Armeria canescens (Host) Boiss. & Plumbaginaceae & $x$ & $\mathrm{x}$ \\
\hline 41 & Arnica montana L. & Arnica montana L. & Asteraceae & $x$ & \\
\hline 42 & Arum maculatum L. & Arum maculatum L. & Araceae & $x$ & $x$ \\
\hline 43 & Aruncus silvester Kostel & $\begin{array}{l}\text { Aruncus dioicus (Walter) } \\
\text { Fernald }\end{array}$ & Rosaceae & $\mathrm{x}$ & \\
\hline 44 & Asarum europaeum L. & Asarum europaeum L. & Aristolochiaceae & $x$ & $\mathrm{x}$ \\
\hline 45 & Asclepias syriaca L. & Asclepias syriaca L. & Asclepiadaceae & $x$ & $\mathrm{x}$ \\
\hline 46 & Asparagus acutifolius L. & Asparagus acutifolius L. & Asparagaceae & $x$ & \\
\hline 47 & Asparagus tenuifolius Lam. & Asparagus tenuifolius Lam. & Asparagaceae & $x$ & $x$ \\
\hline 48 & Asperula tinctoria L. & Asperula tinctoria $\mathrm{L}$. & Rubiaceae & $x$ & $x$ \\
\hline 49 & Asphodelus albus Mill. & Asphodelus albus Mill. & Xanthorrhoeaceae & $x$ & $\mathrm{x}$ \\
\hline 50 & Asphoideline lutea Rchb. & Asphodeline lutea (L.) Rchb. & Xanthorrhoeaceae & $x$ & $x$ \\
\hline 51 & Aster alpinum & Aster alpinus L. & Asteraceae & $x$ & $\mathrm{x}$ \\
\hline 52 & Astragalus glycyphyllos L. & Astragalus glycyphyllos L. & Fabaceae & $x$ & $x$ \\
\hline 53 & Astrantia major L. & Astrantia major $\mathrm{L}$. & Apiaceae & $x$ & $x$ \\
\hline 54 & Atriplex hortense L. & Atriplex hortensis L. & Chenopodiaceae & $x$ & \\
\hline
\end{tabular}




\begin{tabular}{|c|c|c|c|c|c|}
\hline No. & original name on label & valid name & family & HR & ZAHO \\
\hline 55 & Atropa belladonna $\mathrm{L}$. & Atropa belladonna $\mathrm{L}$. & Solanaceae & $x$ & $x$ \\
\hline 56 & Avena barbata Brot. & Avena barbata Pott ex Link & Poaceae & $x$ & \\
\hline 57 & Avena elatior & $\begin{array}{l}\text { Arrhenatherum elatius (L.) } \\
\text { P.Beauv. ex J.Presl \& C.Presl. }\end{array}$ & Poaceae & $\mathrm{x}$ & $\mathrm{x}$ \\
\hline 58 & Ballota & Ballota sp. & Lamiaceae & $x$ & $x$ \\
\hline 59 & Berberis vulgaris L. & Berberis vulgaris L. & Berberidaceae & $x$ & $x$ \\
\hline 60 & Berteroa incana (L.) DC. & Berteroa incana (L.) DC. & Brassicaceae & $\mathrm{x}$ & $x$ \\
\hline 61 & Beta vulgaris L. & Beta vulgaris L. & Amaranthaceae & $\mathrm{x}$ & \\
\hline 62 & Bidens tripartitus L. & Bidens tripartita $\mathrm{L}$. & Asteraceae & $x$ & $x$ \\
\hline 63 & Biscutella laevigata $\mathrm{L}$. & Biscutella laevigata $\mathrm{L}$. & Brassicaceae & $x$ & $x$ \\
\hline 64 & Brassica oleracea L. & Brassica oleracea L. & Brassicaceae & $\mathrm{x}$ & \\
\hline 65 & Briza maxima $\mathrm{L}$. & Briza maxima $\mathrm{L}$. & Poaceae & $\mathrm{x}$ & $x$ \\
\hline 66 & Bromus erectus Huds. & Bromus erectus Huds. & Poaceae & $\mathrm{x}$ & $\mathrm{x}$ \\
\hline 67 & Bromus mollis & Bromus hordeaceus L. & Poaceae & $x$ & $x$ \\
\hline 68 & Bromus sterilis L. & Bromus sterilis L. & Poaceae & $\mathrm{x}$ & $\mathrm{x}$ \\
\hline 69 & Brunella vulgaris L. & Prunella vulgaris L. & Lamiaceae & $x$ & $\mathrm{x}$ \\
\hline 70 & Bunias orientalis L. & Bunias orientalis L. & Brassicaceae & $x$ & $x$ \\
\hline 71 & Buphthalmum salicifolium L. & Buphthalmum salicifolium L. & Asteraceae & $\mathrm{x}$ & $x$ \\
\hline 72 & Calendula officinalis L.J. & Calendula officinalis L.J. & Asteraceae & $\mathrm{x}$ & \\
\hline 73 & Campanula formanekiana & $\begin{array}{l}\text { Campanula formanekiana Degen } \\
\text { \& Dörfl. }\end{array}$ & Campanulaceae & & $\mathrm{x}$ \\
\hline 74 & Campanula persicifolia L.J. & Campanula persicifolia L.J. & Campanulaceae & $x$ & $x$ \\
\hline 75 & Campanula rapunculoides L. & Campanula rapunculoides L. & Campanulaceae & $x$ & $x$ \\
\hline 76 & Cannabis sativa $\mathrm{L}$. & Cannabis sativa L. & Cannabaceae & $x$ & \\
\hline 77 & Capsela bursa-pastoris L. & $\begin{array}{l}\text { Capsella bursa-pastoris (L.) } \\
\text { Medik. }\end{array}$ & Brassicaceae & $\mathrm{x}$ & \\
\hline 78 & Carduus acanthoides L. & Carduus acanthoides L. & Asteraceae & $\mathrm{x}$ & \\
\hline 79 & Carlina aggregata W.K. & $\begin{array}{l}\text { Carlina acaulis subsp. caulescens } \\
\text { (Lam.) Schübl. \& G.Martens }\end{array}$ & Asteraceae & $\mathrm{x}$ & $\mathrm{x}$ \\
\hline 80 & Carpinus betulus L. & Carpinus betulus L. & Corylaceae & $\mathrm{x}$ & $\mathrm{x}$ \\
\hline 81 & Carum carvi $\mathrm{L}$. & Carum carvi $\mathrm{L}$. & Apiaceae & $\mathrm{x}$ & $\mathrm{x}$ \\
\hline 82 & Catalpa bignonioides Walt. & Catalpa bignonioides Walter & Bignoniaceae & $x$ & $x$ \\
\hline 83 & Cedrus Libani Barr. & Cedrus libani A.Rich. & Pinaceae & $\mathrm{x}$ & \\
\hline 84 & Celtis australis $\mathrm{L}$. & Celtis australis $\mathrm{L}$. & Ulmaceae & $\mathrm{x}$ & $x$ \\
\hline 85 & Centaurea Fritschii Hay. & $\begin{array}{l}\text { Centaurea scabiosa ssp. fritschii } \\
\text { (Hayek) Hayek }\end{array}$ & Asteraceae & $\mathrm{x}$ & $x$ \\
\hline 86 & Centaurea leucolepis DC. & $\begin{array}{l}\text { Centaurea deusta Ten. ssp. } \\
\text { concolor (DC.) Hayek }\end{array}$ & Asteraceae & $\mathrm{x}$ & $\mathrm{x}$ \\
\hline 87 & Centaurea rhenana Bor. & Centaurea rhenana Boreau & Asteraceae & $x$ & \\
\hline 88 & Centaurea rupestris L. & Centaurea rupestris L. & Asteraceae & $x$ & $x$ \\
\hline 89 & Centaurea scabiosa $\mathrm{L}$. & Centaurea scabiosa L. & Asteraceae & $x$ & $x$ \\
\hline 90 & Cephalaria leucantha Schrad. & $\begin{array}{l}\text { Cephalaria leucantha (L.) Roem. } \\
\text { et Schult. }\end{array}$ & Dipsacaceae & $\mathrm{x}$ & $\mathrm{x}$ \\
\hline 91 & Ceratonia siliqua $\mathrm{L}$. & Ceratonia siliqua $\mathrm{L}$. & Fabaceae & $\mathrm{x}$ & $x$ \\
\hline 92 & Cercis siliquastrum L. & Cercis siliquastrum L. & Fabaceae & $x$ & $x$ \\
\hline 93 & Chaenorrhinum minus L. & Chaenorhinum minus (L.) Lange & Scrophulariaceae & $x$ & $x$ \\
\hline
\end{tabular}




\begin{tabular}{|c|c|c|c|c|c|}
\hline No. & original name on label & valid name & family & HR & ZAHO \\
\hline 94 & Chaerophyllum aromaticum $\mathrm{L}$. & Chaerophyllum aromaticum L. & Apiaceae & $x$ & $x$ \\
\hline 95 & Chelidonium majus L. & Chelidonium majus L. & Papaveraceae & $\mathrm{x}$ & $x$ \\
\hline 96 & Chenopodium album $\mathrm{L}$. & Chenopodium album $\mathrm{L}$. & Chenopodiaceae & $x$ & $\mathrm{x}$ \\
\hline 97 & Chenopodium polyspermum L. & Chenopodium polyspermum L. & Chenopodiaceae & $\mathrm{x}$ & $x$ \\
\hline 98 & Chondrilla juncea L. & Chondrilla juncea $\mathrm{L}$. & Cichoriaceae & $x$ & $x$ \\
\hline 99 & $\begin{array}{l}\text { Chrysanth. vulgare } \mathrm{L} . \\
\text { Tanacetum vulgare }\end{array}$ & Tanacetum vulgare $\mathrm{L}$. & Asteraceae & $\mathrm{x}$ & $\mathrm{x}$ \\
\hline 100 & $\begin{array}{l}\text { Chrysanthemum } \\
\text { cinerariifolium }\end{array}$ & $\begin{array}{l}\text { Tanacetum cinerariifolium } \\
\text { (Trevir.) Sch.Bip. }\end{array}$ & Asteraceae & $\mathrm{x}$ & $x$ \\
\hline 101 & $\begin{array}{l}\text { Chrysanthemum corymbosum } \\
\text { L. }\end{array}$ & $\begin{array}{l}\text { Tanacetum corymbosum (L.) } \\
\text { Sch.Bip. }\end{array}$ & Asteraceae & $x$ & $x$ \\
\hline 102 & Cicer arietinum $\mathrm{L}$. & Cicer arietinum $\mathrm{L}$. & Fabaceae & $x$ & $x$ \\
\hline 103 & Cichorium intybus L. & Cichorium intybus L. & Cichoriaceae & $\mathrm{x}$ & $x$ \\
\hline 104 & Cinnamomum camphora $\mathrm{N}$. & $\begin{array}{l}\text { Cinnamomum camphora (L.) } \\
\text { J.Presl }\end{array}$ & Lauraceae & & \\
\hline 105 & Cirsium acaule Web. & Cirsium acaulon (L.) Scop. & Asteraceae & $x$ & $x$ \\
\hline 106 & Cirsium eriophorum Scop. & Cirsium eriophorum Scop. & Asteraceae & $x$ & $x$ \\
\hline 107 & Cirsium lanceolatum $\mathrm{L}$. & Cirsium vulgare (Savi) Ten. & Asteraceae & $x$ & \\
\hline 108 & Citrus aurantium L. & Citrus aurantium L. & Rutaceae & $\mathrm{x}$ & \\
\hline 109 & Citrus medica L. & Citrus medica L. & Rutaceae & $\mathrm{x}$ & \\
\hline 110 & Citrus nobilis & Citrus nobilis Lour. & Rutaceae & $\mathrm{x}$ & \\
\hline 111 & Citrus paradisi "Grapefruit" T. & Citrus paradisi Macfad. & Rutaceae & & \\
\hline 112 & Clematis flammula $\mathrm{L}$. & Clematis flammula $\mathrm{L}$. & Ranunculaceae & $x$ & $x$ \\
\hline 113 & Clematis tangutica & $\begin{array}{l}\text { Clematis tangutica (Maxim.) } \\
\text { Korsh. }\end{array}$ & Ranunculaceae & & \\
\hline 114 & Clematis vitalba $\mathrm{L}$. & Clematis vitalba $\mathrm{L}$. & Ranunculaceae & $x$ & $x$ \\
\hline 115 & Cnicus benedictus L. & Centaurea benedicta (L.) L. & Asteraceae & & \\
\hline 116 & Colchicum autumnale L. & Colchicum autumnale L. & Colchicaceae & $x$ & $\mathrm{x}$ \\
\hline 117 & $\begin{array}{l}\text { Colocynthis citrullus (L.) } \\
\text { Fritsch }\end{array}$ & $\begin{array}{l}\text { Citrullus lanatus (Thunb.) } \\
\text { Matsum. \& Nakai }\end{array}$ & Cucurbitaceae & $x$ & \\
\hline 118 & Colutea arborescens L. & Colutea arborescens L. & Fabaceae & $x$ & $\mathrm{x}$ \\
\hline 119 & Conium maculatum $\mathrm{L}$. & Conium maculatum L. & Apiaceae & $x$ & \\
\hline 120 & Convolvulus arvensis $\mathrm{L}$. & Convolvulus arvensis L. & Convolvulaceae & $x$ & $x$ \\
\hline 121 & Convolvus cantabrica L. & Convolvulus cantabrica L. & Convolvulaceae & $\mathrm{x}$ & $\mathrm{x}$ \\
\hline 122 & Coriandrum sativum $\mathrm{L}$. & Coriandrum sativum L. & Apiaceae & $x$ & \\
\hline 123 & Cornus mas L. & Cornus mas L. & Cornaceae & $x$ & $\mathrm{x}$ \\
\hline 124 & $\begin{array}{l}\text { Coronilla emerus ssp. } \\
\text { emeroides L. }\end{array}$ & $\begin{array}{l}\text { Coronilla emerus L. ssp. } \\
\text { emeroides Boiss. et Spruner }\end{array}$ & Fabaceae & $x$ & $x$ \\
\hline 125 & Coronilla varia $\mathrm{L}$. & Securigera varia (L.) Lassen & Fabaceae & $x$ & $\mathrm{x}$ \\
\hline 126 & $\begin{array}{l}\text { Corydalis cava (L.) Schw. et } \\
\text { K. }\end{array}$ & Corydalis cava (L.) Schw. et K. & Fumariaceae & $\mathrm{x}$ & $\mathrm{x}$ \\
\hline 127 & Corylus avellana $\mathrm{L}$. & Corylus avellana $\mathrm{L}$. & Corylaceae & $x$ & $\mathrm{x}$ \\
\hline 128 & Cotoneaster tomentosa Lindl. & $\begin{array}{l}\text { Cotoneaster nebrodensis (Guss.) } \\
\text { K. Koch }\end{array}$ & Rosaceae & $\mathrm{x}$ & $\mathrm{x}$ \\
\hline 129 & Crataegus coccinea L. & Crataegus coccinea $\mathrm{L}$. & Rosaceae & & \\
\hline 130 & $\begin{array}{l}\text { Crataeugs monogyna Jacq., } \\
\text { Crataegus transalpina L. }\end{array}$ & Crataeugs monogyna Jacq. & Rosaceae & $x$ & $\mathrm{x}$ \\
\hline
\end{tabular}




\begin{tabular}{|c|c|c|c|c|c|}
\hline No. & original name on label & valid name & family & HR & $\mathrm{ZAHO}$ \\
\hline 131 & Crepis biennis L. & Crepis biennis Lapeyr. & Cichoriaceae & $x$ & $\mathrm{x}$ \\
\hline 132 & Crepis chondrilloides $\mathrm{L}$. & Crepis chondrilloides Jacq. & Cichoriaceae & $\mathrm{x}$ & $x$ \\
\hline 133 & Crithmum maritimum L. & Crithmum maritimum L. & Apiaceae & $x$ & $x$ \\
\hline 134 & Cucumis melo L. & Cucumis melo L. & Cucurbitaceae & $\mathrm{x}$ & \\
\hline 135 & Cucumis sativus L. & Cucumis sativus L. & Cucurbitaceae & $x$ & \\
\hline 136 & Cucurbita pepo L. & Cucurbita pepo L. & Cucurbitaceae & $x$ & $\mathrm{x}$ \\
\hline 137 & $\begin{array}{l}\text { Cupressus sempervirens v. } \\
\text { stricta } \text { L., Cupressus } \\
\text { sempervirens L. }\end{array}$ & Cupressus sempervirens L. & Cupressaceae & $\mathrm{x}$ & \\
\hline 138 & Cuscuta sp. & Cuscuta sp. & Cuscutaceae & $\mathrm{x}$ & $x$ \\
\hline 139 & Cycas revoluta $\mathrm{L}$. & Cycas revoluta Thunb. & Cycadaceae & & \\
\hline 140 & Cydonia japonica Pers. & $\begin{array}{l}\text { Chaenomeles japonica (Thunb.) } \\
\text { Lindl. ex Spach }\end{array}$ & Rosaceae & $\mathrm{x}$ & $\mathrm{x}$ \\
\hline 141 & Cynanchum vincetoxicum L. & $\begin{array}{l}\text { Vincetoxicum hirundinaria } \\
\text { Medik. }\end{array}$ & Asclepiadaceae & $\mathrm{x}$ & $\mathrm{x}$ \\
\hline 142 & Cynara cardunculus L. & Cynara cardunculus L. & Asteraceae & $x$ & \\
\hline 143 & Cynodon dactylon (L.) Fers. & Cynodon dactylon (L.) Pers. & Poaceae & $x$ & $\mathrm{x}$ \\
\hline 144 & Cynoglossum officinale & Cynoglossum officinale L. & Boraginaceae & $x$ & \\
\hline 145 & Cynosurus cristatus L. & Cynosurus cristatus L. & Poaceae & $\mathrm{x}$ & $\mathrm{x}$ \\
\hline 146 & Cytisus hirsutus L. & Cytisus hirsutus L. & Fabaceae & $\mathrm{x}$ & $x$ \\
\hline 147 & Cytisus laburnum L. & Laburnum anagyroides Medik. & Fabaceae & $\mathrm{x}$ & \\
\hline 148 & Cytisus nigricans L. & $\begin{array}{l}\text { Lembotropis nigricans (L.) } \\
\text { Griseb. }\end{array}$ & Fabaceae & $\mathrm{x}$ & $\mathrm{x}$ \\
\hline 149 & Cytisus supinus L. & Cytisus hirsutus L. & Fabaceae & $\mathrm{x}$ & $x$ \\
\hline 150 & Dactylis glomerata $\mathrm{L}$. & Dactylis glomerata $\mathrm{L}$. & Poaceae & $x$ & $x$ \\
\hline 151 & Daphne laureola L. & Daphne laureola $\mathrm{L}$. & Thymelaeaceae & $x$ & $x$ \\
\hline 152 & Daphne mezereum $\mathrm{L}$. & Daphne mezereum L. & Thymelaeaceae & $x$ & $x$ \\
\hline 153 & Datura stramonium L. & Datura stramonium L. & Solanaceae & $x$ & \\
\hline 154 & Daucus carota $\mathrm{L}$. & Daucus carota $\mathrm{L}$. & Apiaceae & $x$ & \\
\hline 155 & Delphinium consolida L. & Consolida regalis Gray & Ranunculaceae & $x$ & $x$ \\
\hline 156 & Dianthus deltoides L. & Dianthus deltoides L. & Caryophyllaceae & $x$ & $x$ \\
\hline 157 & Dianthus plumarius L. & Dianthus plumarius L. & Caryophyllaceae & $x$ & $x$ \\
\hline 158 & Dictamnus albus L. & Dictamnus albus L. & Rutaceae & $x$ & $\mathrm{x}$ \\
\hline 159 & Digitalis laevigata L. & $\begin{array}{l}\text { Digitalis laevigata Waldst. et } \\
\text { Kit. }\end{array}$ & Scrophulariaceae & $\mathrm{x}$ & $\mathrm{x}$ \\
\hline 160 & Digitalis lanata Ehrh. & Digitalis lanata Ehrh. & Scrophulariaceae & $\mathrm{x}$ & \\
\hline 161 & Digitalis lutea L. & Digitalis lutea L. & Scrophulariaceae & $x$ & \\
\hline 162 & Digitalis purpurea L. & Digitalis purpurea L. & Scrophulariaceae & $\mathrm{x}$ & \\
\hline 163 & Dioscorea caucasica & Dioscorea caucasica Lipsky & Dioscoreaceae & & \\
\hline 164 & Diplotaxis tenuifolia (L.) DC. & Diplotaxis tenuifolia (L.) DC. & Brassicaceae & $x$ & $x$ \\
\hline 165 & Dorycnium herbaceum Vill. & Dorycnium herbaceum Vill. & Fabaceae & $\mathrm{x}$ & $x$ \\
\hline 166 & Draba verna & Erophila verna (L.) DC. & Brassicaceae & $x$ & $x$ \\
\hline 167 & Dryas octopetala L. & Dryas octopetala L. & Rosaceae & $\mathrm{x}$ & $x$ \\
\hline 168 & Ecballium elaterium (L.) Rich. & Ecballium elaterium (L.) A. Rich. & Cucurbitaceae & $x$ & \\
\hline 169 & $\begin{array}{l}\text { Echinochloa crus galli (L.) R. } \\
\text { et. Sch. }\end{array}$ & $\begin{array}{l}\text { Echinochloa crus-galli (L.) P. } \\
\text { Beauv. }\end{array}$ & Poaceae & $\mathrm{x}$ & $x$ \\
\hline
\end{tabular}




\begin{tabular}{|c|c|c|c|c|c|}
\hline No. & original name on label & valid name & family & HR & ZAHO \\
\hline 170 & Echium vulgare L. & Echium vulgare L. & Boraginaceae & $\mathrm{x}$ & $x$ \\
\hline 171 & Edraianthus tenuifolius (W.K.) & $\begin{array}{l}\text { Edraianthus tenuifolius (A.DC.) } \\
\text { A.DC. }\end{array}$ & Campanulaceae & $\mathrm{x}$ & $\mathrm{x}$ \\
\hline 172 & Elaeagnus angustifolia L.J. & Elaeagnus angustifolia L.J. & Elaeagnaceae & $\mathrm{x}$ & \\
\hline 173 & $\begin{array}{l}\text { Epilobium angustifolium } \\
\text { Scop., Epilobium spicatum L. }\end{array}$ & Epilobium angustifolium L. & Onagraceae & $x$ & \\
\hline 174 & $\begin{array}{l}\text { Epilobium rosmarinifolium } \\
\text { Haenke } \mathrm{R} .\end{array}$ & Epilobium dodonaei Vill. & Onagraceae & $\mathrm{x}$ & \\
\hline 175 & Epilobium sp. L. & Epilobium sp. & Onagraceae & $x$ & $x$ \\
\hline 176 & Erigeron canadensis L. & Erigeron canadensis L. & Asteraceae & $x$ & $\mathrm{x}$ \\
\hline 177 & Erodium cicutarium $\mathrm{L}$. & Erodium cicutarium (L.) L'Hér. & Geraniaceae & $x$ & $x$ \\
\hline 178 & Eruca sativa L. & $\begin{array}{l}\text { Eruca vesicaria (L.) Cav. ssp. } \\
\text { sativa (Mill.) Thell. }\end{array}$ & Brassicaceae & $\mathrm{x}$ & \\
\hline 179 & Erythronium dens-canis L. & Erythronium dens-canis L. & Liliaceae & $\mathrm{x}$ & \\
\hline 180 & Euonymus europaea L. & Euonymus europaeus L. & Celastraceae & $x$ & $\mathrm{x}$ \\
\hline 181 & Euonymus japonica Thbg. & Euonymus japonicus Thunb. & Celastraceae & $x$ & $x$ \\
\hline 182 & Eupatorium cannabinum L. & Eupatorium cannabinum L. & Asteraceae & $x$ & $x$ \\
\hline 183 & Euphorbia amygdaloides L. & Euphorbia amygdaloides L. & Euphorbiaceae & $x$ & $x$ \\
\hline 184 & Euphorbia cyparissias L. & Euphorbia cyparissias L. & Euphorbiaceae & $x$ & $\mathrm{x}$ \\
\hline 185 & Euphorbia falcata & Euphorbia falcata L. & Euphorbiaceae & $x$ & \\
\hline 186 & Euphorbia helioscopia L. & Euphorbia helioscopia L. & Euphorbiaceae & $\mathrm{x}$ & $x$ \\
\hline 187 & Euphorbia polychroma Kern. & Euphorbia epithymoides L. & Euphorbiaceae & $\mathrm{x}$ & $x$ \\
\hline 188 & Euphorbia verrucosa L. & Euphorbia verrucosa L. & Euphorbiaceae & $x$ & $\mathrm{x}$ \\
\hline 189 & $\begin{array}{l}\text { Fagopyrum vulgare Hill. } \\
\text { sagittatum Gill. }\end{array}$ & Fagopyrum esculentum Moench & Polygonaceae & $x$ & \\
\hline 190 & Fagus silvatica L. & Fagus sylvatica L. & Fagaceae & $x$ & $\mathrm{x}$ \\
\hline 191 & Ferulago galbanifera Koch. & $\begin{array}{l}\text { Ferulago campestris (Besser) } \\
\text { Grecescu }\end{array}$ & Apiaceae & $x$ & $x$ \\
\hline 192 & Festuca gigantea L. & Festuca gigantea (L.) Vill & Poaceae & $x$ & $x$ \\
\hline 193 & Festuca ovina L. & Festuca ovina L. & Poaceae & $\mathrm{x}$ & $\mathrm{x}$ \\
\hline 194 & Festuca pratensis Huds. & Festuca pratensis Huds. & Poaceae & $x$ & $x$ \\
\hline 195 & Filago arvensis $\mathrm{L}$. & Filago arvensis L. & Asteraceae & $x$ & $x$ \\
\hline 196 & Filipendula hexapetala Gilib. & Filipendula vulgaris Moench & Rosaceae & $\mathrm{x}$ & $x$ \\
\hline 197 & Filipendula ulmaria Maxim. & Filipendula ulmaria (L.) Maxim. & Rosaceae & $\mathrm{x}$ & $x$ \\
\hline 198 & Foeniculum vulgare Mill. & Foeniculum vulgare Mill. & Apiaceae & $x$ & \\
\hline 199 & Fraxinus ornus L. & Fraxinus ornus L. & Oleaceae & $x$ & $\mathrm{x}$ \\
\hline 200 & Fumana procumbens & $\begin{array}{l}\text { Fumana procumbens (Dunal) } \\
\text { Gren. \& Godr. }\end{array}$ & Cistaceae & $\mathrm{x}$ & \\
\hline 201 & Galega officinalis L. & Galega officinalis L. & Fabaceae & $x$ & $x$ \\
\hline 202 & Galeopsis speciosa Mill. & Galeopsis speciosa Mill. & Lamiaceae & $\mathrm{x}$ & $x$ \\
\hline 203 & Galinsoga parviflora Carvan. & Galinsoga parviflora Cav. & Asteraceae & $x$ & \\
\hline 204 & Galium vernum $\mathrm{L}$. & Cruciata glabra (L.) Opiz & Rubiaceae & $x$ & $\mathrm{x}$ \\
\hline 205 & Galtonia candicans Decaisne J. & $\begin{array}{l}\text { Ornithogalum candicans (Baker) } \\
\text { J.C.Manning \& Goldblatt }\end{array}$ & Asparagaceae & & \\
\hline 206 & Gentiana anisodonta Borb. & $\begin{array}{l}\text { Gentianella anisodonta (Borbás) } \\
\text { Á. Löve et D. Löve }\end{array}$ & Gentianaceae & $\mathrm{x}$ & $\mathrm{x}$ \\
\hline 207 & Gentiana asclepiadea L. & Gentiana asclepiadea L. & Gentianaceae & $x$ & $\mathrm{x}$ \\
\hline
\end{tabular}




\begin{tabular}{|c|c|c|c|c|c|}
\hline No. & original name on label & valid name & family & HR & $\mathrm{ZAHO}$ \\
\hline 208 & Geranium robertianum $\mathrm{L}$. & Geranium robertianum L. & Geraniaceae & $x$ & $\mathrm{x}$ \\
\hline 209 & Geum urbanum $\mathrm{L}$. & Geum urbanum L. & Rosaceae & $\mathrm{x}$ & $\mathrm{x}$ \\
\hline 210 & Gladiolus illyricus Koch. & $\begin{array}{l}\text { Gladiolus illyricus W. D. J. } \\
\text { Koch. }\end{array}$ & Iridaceae & $\mathrm{x}$ & $\mathrm{x}$ \\
\hline 211 & Glaucium luteum & Glaucium flavum Crantz & Papaveraceae & $x$ & $x$ \\
\hline 212 & Gleditschia triacanthos L. & Gleditsia triacanthos L. & Fabaceae & $\mathrm{x}$ & \\
\hline 213 & Globularia bellidifolia Ten. & $\begin{array}{l}\text { Globularia cordifolia L. ssp. } \\
\text { bellidifolia (Ten.) Wettst. }\end{array}$ & Globulariaceae & $\mathrm{x}$ & $\mathrm{x}$ \\
\hline 214 & Globularia Willkommii Nym. & Globularia punctata Lapeyr. & Globulariaceae & $x$ & $x$ \\
\hline 215 & Gomphocarpus fruticosus L. & $\begin{array}{l}\text { Gomphocarpus fruticosus (L.) } \\
\text { W.T.Aiton }\end{array}$ & Asclepiadaceae & $\mathrm{x}$ & \\
\hline 216 & Gossypium herbaceus L. & Gossypium herbaceum L. & Malvaceae & $x$ & \\
\hline 217 & Hedera helix $\mathrm{L}$. & Hedera helix L. & Araliaceae & $x$ & $x$ \\
\hline 218 & Helianthemum obscurum & $\begin{array}{l}\text { Helianthemum nummularium } \\
\text { (L.) Mill. ssp. obscurum } \\
\text { (Čelak.) Holub }\end{array}$ & Cistaceae & $\mathrm{x}$ & $\mathrm{x}$ \\
\hline 219 & Helianthus annua L. & Helianthus annuus L. & Asteraceae & $\mathrm{x}$ & \\
\hline 220 & Helichrysum italicum Guss. & $\begin{array}{l}\text { Helichrysum italicum (Roth) } \\
\text { G.Don }\end{array}$ & Asteraceae & $\mathrm{x}$ & $\mathrm{x}$ \\
\hline 221 & Heracleum sphondylium L. & Heracleum sphondylium L. & Apiaceae & $\mathrm{x}$ & $x$ \\
\hline 222 & Hibiscus syriacus L. & Hibiscus syriacus L. & Malvaceae & $\mathrm{x}$ & \\
\hline 223 & Hibiscus trionum L. & Hibiscus trionum L. & Malvaceae & $x$ & $\mathrm{x}$ \\
\hline 224 & Hieracium pillosella $\mathrm{L}$. & Hieracium pilosella $\mathrm{L}$. & Cichoriaceae & $x$ & $x$ \\
\hline 225 & Holcus lanataum & Holcus lanatus L. & Poaceae & $x$ & $\mathrm{x}$ \\
\hline 226 & $\begin{array}{l}\text { Homogyne silvestris (Scop.) } \\
\text { Cass. }\end{array}$ & $\begin{array}{l}\text { Homogyne sylvestris (Jacq.) } \\
\text { Cass. }\end{array}$ & Asteraceae & $\mathrm{x}$ & $\mathrm{x}$ \\
\hline 227 & Hordeum sativum L. & Hordeum vulgare $\mathrm{L}$. & Poaceae & $x$ & \\
\hline 228 & Humulus lupulus L. & Humulus lupulus L. & Cannabaceae & $x$ & $x$ \\
\hline 229 & Hyoscyamus niger & Hyoscyamus niger L. & Solanaceae & $x$ & $\mathrm{x}$ \\
\hline 230 & Hypericum montanum L. & Hypericum montanum L. & Clusiaceae & $x$ & $x$ \\
\hline 231 & Hypericum perforatum $\mathrm{L}$. & Hypericum perforatum $\mathrm{L}$. & Hypericaceae & $x$ & $x$ \\
\hline 232 & Hypochoeris maculata L. & Hypochaeris maculata L. & Cichoriaceae & $x$ & $x$ \\
\hline 233 & Hypochoeris radicata $\mathrm{L}$. & Hypochaeris radicata $\mathrm{L}$. & Cichoriaceae & $x$ & $x$ \\
\hline 234 & Hyssopus officinalis L. & Hyssopus officinalis L. & Lamiaceae & $x$ & $x$ \\
\hline 235 & Impatiens noli-tangere L. & Impatiens noli-tangere L. & Balsaminaceae & $x$ & $x$ \\
\hline 236 & Inula britannica $\mathrm{L}$. & Inula britannica L. & Asteraceae & $x$ & $x$ \\
\hline 237 & Inula conyza DC. & Inula conyza (Griess.) DC. & Asteraceae & $x$ & $x$ \\
\hline 238 & Inula helenium $\mathrm{L}$. & Inula helenium L. & Asteraceae & $x$ & \\
\hline 239 & Inula hirta L. & Inula hirta L. & Asteraceae & $x$ & $x$ \\
\hline 240 & Iris illyrica Tomm. & Iris illyrica Tomm. & Iridaceae & $x$ & $x$ \\
\hline 241 & Iris pseudacorus L. & Iris pseudacorus L. & Iridaceae & $x$ & $x$ \\
\hline 242 & Iris sibirica L. & Iris sibirica L. & Iridaceae & $x$ & $x$ \\
\hline 243 & Isatis tinctoria $\mathrm{L}$. & Isatis tinctoria $\mathrm{L}$. & Brassicaceae & $x$ & \\
\hline 244 & Jurinea mollis L. & Jurinea mollis (L.) Rchb. & Asteraceae & $x$ & $x$ \\
\hline 245 & Kickxia spurea (L.) Dum. & Kickxia spuria (L.) Dumort. & Scrophulariaceae & $x$ & $x$ \\
\hline 246 & Knautia arvensis $\mathrm{L}$. & Knautia arvensis (L.) Coult. & Dipsacaceae & $x$ & $x$ \\
\hline
\end{tabular}




\begin{tabular}{|c|c|c|c|c|c|}
\hline No. & original name on label & valid name & family & HR & $\mathrm{ZAHO}$ \\
\hline 247 & Kniphofia uvaria Hook. & Kniphofia uvaria (L.) Oken & Asphodelaceae & & \\
\hline 248 & Kochia scoparia (L.) Schrad.J. & Bassia scoparia (L.) A. J. Scott & Chenopodiaceae & $x$ & \\
\hline 249 & $\begin{array}{l}\text { Koelreuteria paniculata } \\
\text { Laxm.A. }\end{array}$ & Koelreuteria paniculata Laxm. & Sapindaceae & $\mathrm{x}$ & \\
\hline 250 & Lactuca muralis Gartn. & Lactuca muralis (L.) Fresen. & Cichoriaceae & $x$ & \\
\hline 251 & Lactuca serriola L. & Lactuca serriola L. & Cichoriaceae & $x$ & \\
\hline 252 & Lamium purpureum L. & Lamium purpureum L. & Lamiaceae & $\mathrm{x}$ & $\mathrm{x}$ \\
\hline 253 & Lapsana communis L. & Lapsana communis L. & Cichoriaceae & $x$ & $x$ \\
\hline 254 & Laserpitium siler $\mathrm{L}$. & Laserpitium siler $\mathrm{L}$. & Apiaceae & $x$ & $x$ \\
\hline 255 & Lathyrus aphaca L. & Lathyrus aphaca L. & Fabaceae & $x$ & $x$ \\
\hline 256 & Lathyrus niger L. & Lathyrus niger L. (Bernh) & Fabaceae & $x$ & $x$ \\
\hline 257 & Lathyrus satious L. & Lathyrus sativus L. & Fabaceae & $\mathrm{x}$ & $x$ \\
\hline 258 & Lathyrus silvestris & Lathyrus sylvestris L. & Fabaceae & $x$ & $\mathrm{x}$ \\
\hline 259 & Lathyrus sp. & Lathyrus sp. & Fabaceae & $x$ & $\mathrm{x}$ \\
\hline 260 & Lathyrus vernus Bernh. & Lathyrus vernus (L.) Bernh. & Fabaceae & $x$ & $x$ \\
\hline 261 & Laurus nobilis L. & Laurus nobilis L. & Lauraceae & $\mathrm{x}$ & $x$ \\
\hline 262 & Lavandula vera DC. & $\begin{array}{l}\text { Lavandula angustifolia subsp. } \\
\text { pyrenaica (DC.) Guinea }\end{array}$ & Lamiaceae & & \\
\hline 263 & Leontodon autumnalis L. & Leontodon autumnalis L. & Cichoriaceae & $x$ & $x$ \\
\hline 264 & Leontodon danubialis Jacqu. & $\begin{array}{l}\text { Leontodon hispidus L. ssp. } \\
\text { danubialis (Jacq) Simonk. }\end{array}$ & Cichoriaceae & $x$ & $x$ \\
\hline 265 & Lepidium campestre (L.) R. Br. & Lepidium campestre (L.) R. Br. & Brassicaceae & $x$ & \\
\hline 266 & Lepidium sativum $\mathrm{L}$. & Lepidium sativum $\mathrm{L}$. & Brassicaceae & $x$ & \\
\hline 267 & Leucanthemum Liburnicum L. & $\begin{array}{l}\text { Leucanthemum atratum (Jacq.) } \\
\text { DC. ssp. platylepis (Borbás) } \\
\text { Heywood }\end{array}$ & Asteraceae & $\mathrm{x}$ & $\mathrm{x}$ \\
\hline 268 & Leucoium aestivum $\mathrm{L}$. & Leucojum aestioum L. & Amaryllidaceae & $x$ & $\mathrm{x}$ \\
\hline 269 & Levisticum officinale Koch. & $\begin{array}{l}\text { Levisticum officinale W. D. J. } \\
\text { Koch }\end{array}$ & Apiaceae & $\mathrm{x}$ & \\
\hline 270 & Libanotis montana $\mathrm{Cr}$. & $\begin{array}{l}\text { Seseli libanotis (L.) W. D. J. } \\
\text { Koch }\end{array}$ & Apiaceae & $\mathrm{x}$ & $\mathrm{x}$ \\
\hline 271 & Ligularia vulgaris & Ligularia sibirica (L.) Cass. & Asteraceae & $x$ & \\
\hline 272 & Ligustrum vulgare L. & Ligustrum vulgare L. & Oleaceae & $x$ & $x$ \\
\hline 273 & Lilium martagon L. & Lilium martagon L. & Liliaceae & $x$ & $x$ \\
\hline 274 & Linum catharticum $\mathrm{L}$. & Linum catharticum $\mathrm{L}$. & Linaceae & $x$ & $\mathrm{x}$ \\
\hline 275 & Linum usitatissimum L. & Linum usitatissimum L. & Linaceae & $x$ & $\mathrm{x}$ \\
\hline 276 & Lithospermum arvense L. & Lithospermum arvense L. & Boraginaceae & $x$ & $x$ \\
\hline 277 & $\begin{array}{l}\text { Lolium italicum (č.sel.), } \\
\text { Lolium westwoldicum }\end{array}$ & Lolium multiflorum Lam. & Poaceae & $x$ & \\
\hline 278 & Lolium perenne L. & Lolium perenne L. & Poaceae & $x$ & $\mathrm{x}$ \\
\hline 279 & Lonicera alpigena L. & Lonicera alpigena L. & Caprifoliaceae & $x$ & $x$ \\
\hline 280 & Lotus corniculatus L. & Lotus corniculatus L. & Fabaceae & $x$ & $x$ \\
\hline 281 & Lunaria rediviva L. & Lunaria rediviva $\mathrm{L}$. & Brassicaceae & $x$ & $x$ \\
\hline 282 & Lupinus albus L. & Lupinus albus L. & Fabaceae & $x$ & \\
\hline 283 & Lycopus europaeus L. & Lycopus europaeus L. & Lamiaceae & $x$ & $\mathrm{x}$ \\
\hline 284 & Majorana hortensis Mnch. & Origanum majorana L. & Lamiaceae & $x$ & \\
\hline
\end{tabular}




\begin{tabular}{|c|c|c|c|c|c|}
\hline No. & original name on label & valid name & family & HR & $\mathrm{ZAHO}$ \\
\hline 285 & $\begin{array}{l}\text { Malus pumila Mill. } \\
\text { ("budimka"; "citronka"; } \\
\text { "delicious",;"pogačnica"; } \\
\text { "reneta";"srčika";"tafetica") }\end{array}$ & Malus pumila Mill. & Rosaceae & $\mathrm{x}$ & \\
\hline 286 & $\begin{array}{l}\text { Malva silvestris L., Malva } \\
\text { mauritiana L. }\end{array}$ & Malva sylvestris $\mathrm{L}$. & Malvaceae & $\mathrm{x}$ & $\mathrm{x}$ \\
\hline 287 & Marrubium vulgare L. & Marrubium vulgare $\mathrm{L}$. & Lamiaceae & $\mathrm{x}$ & \\
\hline 288 & Matricaria discoidea DC. & Matricaria discoidea DC. & Asteraceae & $x$ & \\
\hline 289 & Matricaria inodora L. & Matricaria perforata Mérat & Asteraceae & $\mathrm{x}$ & $\mathrm{x}$ \\
\hline 290 & Medicago lupulina L. & Medicago lupulina L. & Fabaceae & $x$ & $x$ \\
\hline 291 & Medicago orbicularis All. & Medicago orbicularis (L.) Bartal. & Fabaceae & $x$ & $x$ \\
\hline 292 & Medicago sativa L. & Medicago sativa L. & Fabaceae & $\mathrm{x}$ & $\mathrm{x}$ \\
\hline 293 & Medicago tuberculata Willd. & $\begin{array}{l}\text { Medicago tuberculata (Retz.) } \\
\text { Willd. }\end{array}$ & Fabaceae & $\mathrm{x}$ & $\mathrm{x}$ \\
\hline 294 & $\begin{array}{l}\text { Melandryum album (Mill.) } \\
\text { Garcke. }\end{array}$ & $\begin{array}{l}\text { Silene latifolia Poir. ssp. alba } \\
\text { (Mill.) Greuter et Bourdet }\end{array}$ & Caryophyllaceae & $\mathrm{x}$ & \\
\hline 295 & Melandryum silvestre Rohl. & Silene dioica (L.) Clairv. & Caryophyllaceae & $\mathrm{x}$ & $x$ \\
\hline 296 & Melia azedarach $\mathrm{L}$. & Melia azedarach L. & Meliaceae & $\mathrm{x}$ & \\
\hline 297 & Melica ciliata L. & Melica ciliata L. & Poaceae & $x$ & $x$ \\
\hline 298 & Melilotus alba L. & Melilotus albus Medik. & Fabaceae & $x$ & $x$ \\
\hline 299 & Melissa officinalis L. & Melissa officinalis L. & Lamiaceae & $\mathrm{x}$ & $x$ \\
\hline 300 & Mirabilis jalapa L. & Mirabilis jalapa L. & Nyctaginaceae & $x$ & \\
\hline 301 & Muscari comosum L. & Muscari comosum (L.) Mill. & Asparagaceae & $x$ & $x$ \\
\hline 302 & Myrtus communis Mill. & Myrtus communis L. & Myrtaceae & $x$ & $x$ \\
\hline 303 & Nerium oleander L. & Nerium oleander L. & Apocynaceae & $x$ & $x$ \\
\hline 304 & Nicandra physaloides Gartn. & $\begin{array}{l}\text { Nicandra physalodes (L.) } \\
\text { Gaertn. }\end{array}$ & Solanaceae & $\mathrm{x}$ & \\
\hline 305 & Nicotiana tabacum L. & Nicotiana tabacum L. & Solanaceae & $x$ & \\
\hline 306 & Nigella arvensis L. & Nigella arvensis L. & Ranunculaceae & $x$ & $\mathrm{x}$ \\
\hline 307 & Nigella damascena $\mathrm{L}$. & Nigella damascena $\mathrm{L}$. & Ranunculaceae & $x$ & $x$ \\
\hline 308 & Ocimum basilicum $\mathrm{L}$. & Ocimum basilicum L. & Lamiaceae & $x$ & \\
\hline 309 & Oenothera biennis L. & Oenothera biennis L. & Onagraceae & $x$ & \\
\hline 310 & Olea europaea & Olea europaea L. & Oleaceae & $x$ & $x$ \\
\hline 311 & Ononis spinosa $\mathrm{L}$. & Ononis spinosa $\mathrm{L}$. & Fabaceae & $x$ & $x$ \\
\hline 312 & Orlaya grandiflora Hoffm. & Orlaya grandiflora (L.) Hoffm. & Apiaceae & $x$ & $x$ \\
\hline 313 & Ornithogalum umbellatum L. & Ornithogalum umbellatum L. & Asparagaceae & $\mathrm{x}$ & $x$ \\
\hline 314 & Oryza sativa L. & Oryza sativa L. & Poaceae & $x$ & \\
\hline 315 & Ostrya carpinifolia Scop. & Ostrya carpinifolia Scop. & Corylaceae & $x$ & $x$ \\
\hline 316 & Osyris alba L. & Osyris alba $\mathrm{L}$. & Santalaceae & $x$ & $x$ \\
\hline 317 & Oxalis stricta $\mathrm{L}$. & Oxalis stricta $\mathrm{L}$. & Oxalidaceae & $x$ & $x$ \\
\hline 318 & Paeonia corallina Retz. & Paeonia mascula (L.) Mill. & Paeoniaceae & $x$ & $x$ \\
\hline 319 & Paliurus spina Christi Mill. & Paliurus spina-christi Mill. & Rhamnaceae & $x$ & $x$ \\
\hline 320 & Panicum capillare L. & Panicum capillare L. & Poaceae & $x$ & $\mathrm{x}$ \\
\hline 321 & Panicum miliaceum L. & Panicum miliaceum L. & Poaceae & $x$ & $x$ \\
\hline 322 & Papaver orientale L. & Papaver orientale L. & Papaveraceae & $x$ & \\
\hline 323 & Papaver rhoeas L. & Papaver rhoeas L. & Papaveraceae & $x$ & $x$ \\
\hline
\end{tabular}




\begin{tabular}{|c|c|c|c|c|c|}
\hline No. & original name on label & valid name & family & HR & ZAHO \\
\hline 324 & Papaver somniferum $\mathrm{L}$. & Papaver somniferum L. & Papaveraceae & $x$ & \\
\hline 325 & Paris quadrifolia L. & Paris quadrifolia L. & Melanthiaceae & $x$ & $x$ \\
\hline 326 & Parnassia palustris L. & Parnassia palustris L. & Parnassiaceae & $x$ & $\mathrm{x}$ \\
\hline 327 & Pastinaca sativa L. & Pastinaca sativa L. & Apiaceae & $\mathrm{x}$ & $\mathrm{x}$ \\
\hline 328 & Peltaria alliacea Jacq. & Peltaria alliacea Jacq. & Brassicaceae & $\mathrm{x}$ & \\
\hline 329 & Petasites albus (L.) Gartn. & Petasites albus (L.) Gaertn. & Asteraceae & $x$ & $x$ \\
\hline 330 & Petroselinum hortense Hoffm. & $\begin{array}{l}\text { Petroselinum crispum (Mill.) } \\
\text { Fuss }\end{array}$ & Apiaceae & $\mathrm{x}$ & \\
\hline 331 & $\begin{array}{l}\text { Peucedanum oreoselinum (L.) } \\
\text { Mnch. }\end{array}$ & $\begin{array}{l}\text { Peucedanum oreoselinum (L.) } \\
\text { Moench. }\end{array}$ & Apiaceae & $\mathrm{x}$ & $\mathrm{x}$ \\
\hline 332 & Phacelia tanacetifolia Benth. & Phacelia tanacetifolia Benth. & Hydrophyllaceae & $x$ & \\
\hline 333 & Phleum pratense $\mathrm{L}$. & Phleum pratense $\mathrm{L}$. & Poaceae & $\mathrm{x}$ & $\mathrm{x}$ \\
\hline 334 & Phoenix canariensis L. & Phoenix canariensis Chabaud & Arecaceae & $\mathrm{x}$ & \\
\hline 335 & Physalis alkekengi $\mathrm{L}$. & Physalis alkekengi $\mathrm{L}$. & Solanaceae & $\mathrm{x}$ & $\mathrm{x}$ \\
\hline 336 & Phytolacca americana $\mathrm{L}$. & Phytolacca americana L. & Phytolaccaceae & $\mathrm{x}$ & $\mathrm{x}$ \\
\hline 337 & Picea excelsa Lk. & Picea abies (L.) H. Karst. & Pinaceae & $\mathrm{x}$ & \\
\hline 338 & Picris hieracioides & Picris hieracioides Sibth. \& Sm. & Cichoriaceae & $\mathrm{x}$ & \\
\hline 339 & $\begin{array}{l}\begin{array}{l}\text { Pinus communis L. } \\
\text { "citronka" }\end{array} \\
\end{array}$ & Pyrus communis L. & Rosaceae & $\mathrm{x}$ & \\
\hline 340 & Pinus halepensis Mill. & Pinus halepensis Mill. & Pinaceae & $\mathrm{x}$ & \\
\hline 341 & Pinus peuce (Perister) & Pinus peuce Griseb. & Pinaceae & & $x$ \\
\hline 342 & Pinus pinea $\mathrm{L}$. & Pinus pinea $\mathrm{L}$. & Pinaceae & $x$ & \\
\hline 343 & Pistacia lentiscus L. & Pistacia lentiscus L. & Anacardiaceae & $\mathrm{x}$ & $\mathrm{x}$ \\
\hline 344 & $\begin{array}{l}\text { Pisum arvensis L., Pisum } \\
\text { sativum L. }\end{array}$ & Pisum satioum $\mathrm{L}$ & Fabaceae & $\mathrm{x}$ & \\
\hline 345 & $\begin{array}{l}\text { Pittosporum tobira (Thbg.) } \\
\text { Ait. }\end{array}$ & $\begin{array}{l}\text { Pittosporum tobira (Thunb.) } \\
\text { W.T.Aiton }\end{array}$ & Pittosporaceae & $x$ & $x$ \\
\hline 346 & Plantago carinata Schrad. & Plantago subulata $\mathrm{L}$. & Plantaginaceae & $\mathrm{x}$ & $x$ \\
\hline 347 & Plantago indica $\mathrm{L}$. & Plantago indica $\mathrm{L}$. & Plantaginaceae & $\mathrm{x}$ & $x$ \\
\hline 348 & Plantago intermedia & $\begin{array}{l}\text { Plantago major ssp. intermedia } \\
\text { (Gilib.) Lange }\end{array}$ & Plantaginaceae & $\mathrm{x}$ & \\
\hline 349 & Plantago lanceolata $\mathrm{L}$. & Plantago lanceolata $\mathrm{L}$. & Plantaginaceae & $\mathrm{x}$ & $x$ \\
\hline 350 & Plantago major $\mathrm{L}$. & Plantago major L. & Plantaginaceae & $x$ & $x$ \\
\hline 351 & Platanus orientalis L. & Platanus orientalis $\mathrm{L}$. & Platanaceae & $\mathrm{x}$ & \\
\hline 352 & Poa nemoralis L. & Poa nemoralis L. & Poaceae & $\mathrm{x}$ & $x$ \\
\hline 353 & Poa palustris L. serotina Ehr. & Poa palustris L. & Poaceae & $\mathrm{x}$ & $x$ \\
\hline 354 & Poa pratensis L. & Poa pratensis L. & Poaceae & $\mathrm{x}$ & $x$ \\
\hline 355 & Poa trivialis & Poa trivialis $\mathrm{L}$. & Poaceae & $\mathrm{x}$ & $\mathrm{x}$ \\
\hline 356 & Polygonum lapathifolium L. & Polygonum lapathifolium L. & Polygonaceae & $\mathrm{x}$ & $x$ \\
\hline 357 & Portulaca oleracea L. & Portulaca oleracea L. & Portulacaceae & $\mathrm{x}$ & \\
\hline 358 & Prenanthes purpurea & Prenanthes purpurea $\mathrm{L}$. & Cichoriaceae & $\mathrm{x}$ & $x$ \\
\hline 359 & Primula columnae Ten. & $\begin{array}{l}\text { Primula veris L. ssp. columnae } \\
\text { (Ten.) Lüdi }\end{array}$ & Primulaceae & $\mathrm{x}$ & $\mathrm{x}$ \\
\hline 360 & Prunella laciniata $\mathrm{L}$. & Prunella laciniata (L.) L. & Lamiaceae & $\mathrm{x}$ & $x$ \\
\hline 361 & Prunus armeniaca L.J. & Prunus armeniaca L. & Rosaceae & $\mathrm{x}$ & \\
\hline 362 & Prunus communis J. (L.) & $\begin{array}{l}\text { Prunus dulcis (Mill.) D. A. } \\
\text { Webb }\end{array}$ & Rosaceae & $x$ & \\
\hline
\end{tabular}




\begin{tabular}{|c|c|c|c|c|c|}
\hline No. & original name on label & valid name & family & HR & $\mathrm{ZAHO}$ \\
\hline 363 & Prunus spinosa L. & Prunus spinosa $\mathrm{L}$. & Rosaceae & $\mathrm{x}$ & $\mathrm{x}$ \\
\hline 364 & Ptelea trifoliata $\mathrm{L}$. & Ptelea trifoliata $\mathrm{L}$. & Rutaceae & & \\
\hline 365 & Punica granatum L. & Punica granatum L. & Punicaceae & $\mathrm{x}$ & $\mathrm{x}$ \\
\hline 366 & Pyracantha coccinea Rom.j. & Pyracantha coccinea M.Roem & Rosaceae & $x$ & \\
\hline 367 & Quercus cerris L. & Quercus cerris L. & Fagaceae & $x$ & $x$ \\
\hline 368 & Quercus conferta Kit. & Quercus frainetto Ten. & Fagaceae & $\mathrm{x}$ & $\mathrm{x}$ \\
\hline 369 & Quercus ilex L. & Quercus ilex L. & Fagaceae & $x$ & $x$ \\
\hline 370 & Quercus palustris & Quercus palustris Münchh. & Fagaceae & $x$ & $x$ \\
\hline 371 & Ranunculus acer L. & Ranunculus acris L. & Ranunculaceae & $x$ & $x$ \\
\hline 372 & Ranunculus sardous Cr. & Ranunculus sardous Crantz & Ranunculaceae & $\mathrm{x}$ & $\mathrm{x}$ \\
\hline 373 & Reseda lutea L. & Reseda lutea L. & Resedaceae & $\mathrm{x}$ & $x$ \\
\hline 374 & Rheum palmatum L. & Rheum palmatum L. & Polygonaceae & & \\
\hline 375 & Ricinus communis $\mathrm{L}$. & Ricinus communis L. & Euphorbiaceae & $\mathrm{x}$ & \\
\hline 376 & Robinia pseudoacacia $\mathrm{L}$. & Robinia pseudoacacia L. & Fabaceae & $x$ & $\mathrm{x}$ \\
\hline 377 & Roripa silvestris Bess. & Rorippa sylvestris (L.) Besser & Brassicaceae & $x$ & $\mathrm{x}$ \\
\hline 378 & Rubia peregrina L. & Rubia peregrina $\mathrm{L}$. & Rubiaceae & $\mathrm{x}$ & $\mathrm{x}$ \\
\hline 379 & Rumex acetosella $\mathrm{L}$. & Rumex acetosella $\mathrm{L}$. & Polygonaceae & $x$ & $\mathrm{x}$ \\
\hline 380 & Rumex obtusifolius L. & Rumex obtusifolius L. & Polygonaceae & $x$ & $x$ \\
\hline 381 & Rumex patientia L. & Rumex patientia L. & Polygonaceae & $x$ & \\
\hline 382 & Rumex scutatus L. & Rumex scutatus L. & Polygonaceae & $x$ & $x$ \\
\hline 383 & Ruscus hypoglossum & Ruscus hypoglossum L. & Asparagaceae & $\mathrm{x}$ & $x$ \\
\hline 384 & $\begin{array}{l}\text { Ruta graveolens L., Ruta } \\
\text { divaricata Ten. }\end{array}$ & Ruta graveolens L. & Rutaceae & $\mathrm{x}$ & $\mathrm{x}$ \\
\hline 385 & Salvia officinalis L. & Salvia officinalis $\mathrm{L}$. & Lamiaceae & $x$ & $\mathrm{x}$ \\
\hline 386 & Salvia pratensis $\mathrm{L}$. & Salvia pratensis $\mathrm{L}$. & Lamiaceae & $x$ & $x$ \\
\hline 387 & Salvia sclarea L. & Salvia sclarea L. & Lamiaceae & $\mathrm{x}$ & $\mathrm{x}$ \\
\hline 388 & Salvia verticillata $\mathrm{L}$. & Salvia verticillata $\mathrm{L}$. & Lamiaceae & $x$ & $x$ \\
\hline 389 & Sambucus nigra L. & Sambucus nigra L. & Caprifoliaceae & $x$ & $\mathrm{x}$ \\
\hline 390 & Sambucus racemosa $\mathrm{L}$. & Sambucus racemosa $\mathrm{L}$. & Caprifoliaceae & $x$ & $x$ \\
\hline 391 & Sanguisorba muricata (Spach.) & $\begin{array}{l}\text { Sanguisorba minor Scop. ssp. } \\
\text { muricata Briq. }\end{array}$ & Rosaceae & $\mathrm{x}$ & $\mathrm{x}$ \\
\hline 392 & Saponaria officinalis & Saponaria officinalis L. & Caryophyllaceae & $x$ & $x$ \\
\hline 393 & Satureja vulgaris Fritsch. & Clinopodium vulgare $\mathrm{L}$. & Lamiaceae & $x$ & $x$ \\
\hline 394 & Scabiosa graminifolia $\mathrm{L}$. & Scabiosa graminifolia $\mathrm{L}$. & Dipsacaceae & $x$ & $x$ \\
\hline 395 & Scilla pratensis Waldst. & $\begin{array}{l}\text { Chouardia litardierei (Breistr.) } \\
\text { Speta }\end{array}$ & Asparagaceae & $\mathrm{x}$ & $\mathrm{x}$ \\
\hline 396 & Scopolia carniolica Jacq. & Scopolia carniolica Jacq. & Solanaceae & $x$ & $x$ \\
\hline 397 & Scorzonera villosa Scop. & Scorzonera villosa Scop. & Cichoriaceae & $x$ & $\mathrm{x}$ \\
\hline 398 & Scrophularia canina L. & Scrophularia canina L. & Scrophulariaceae & $x$ & $x$ \\
\hline 399 & Secale cereale $\mathrm{L}$. & Secale cereale L. & Poaceae & $x$ & \\
\hline 400 & Senecio jacobaea L. & Senecio jacobaea L. & Asteraceae & $x$ & $x$ \\
\hline 401 & Senecio nemorensis L. & Senecio nemorensis L. & Asteraceae & $x$ & $x$ \\
\hline 402 & Senecio umbrosus W.K.B. & Senecio umbrosus Waldst. \& Kit. & Asteraceae & $x$ & $x$ \\
\hline 403 & Senecio vulgaris L. & Senecio vulgaris $\mathrm{L}$. & Asteraceae & $\mathrm{x}$ & \\
\hline 404 & Sesanum indicum L.J. & Sesamum indicum L. & Pedaliaceae & & \\
\hline
\end{tabular}




\begin{tabular}{|c|c|c|c|c|c|}
\hline No. & original name on label & valid name & family & HR & $\mathrm{ZAHO}$ \\
\hline 405 & Setaria glauca (L.) R. et. Sch. & Pennisetum glaucum (L.) R. Br. & Poaceae & $\mathrm{x}$ & $\mathrm{x}$ \\
\hline 406 & Setaria italica & Setaria italica (L.) P. Beauv. & Poaceae & $\mathrm{x}$ & \\
\hline 407 & Silene sp. & Silene sp. & Caryophyllaceae & $\mathrm{x}$ & $\mathrm{x}$ \\
\hline 408 & Sinapis alba L. & Sinapis alba L. & Brassicaceae & $x$ & \\
\hline 409 & Sinapis arvensis $\mathrm{L}$. & Sinapis arvensis L. & Brassicaceae & $\mathrm{x}$ & \\
\hline 410 & Sinapis sp. & Sinapis sp. & Brassicaceae & $\mathrm{x}$ & \\
\hline 411 & $\begin{array}{l}\text { Sisymbrium officinale (L.) } \\
\text { Scop. }\end{array}$ & Sisymbrium officinale (L.) Scop. & Brassicaceae & $\mathrm{x}$ & $\mathrm{x}$ \\
\hline 412 & Sisymbrium strictissimum L. & Sisymbrium strictissimum L. & Brassicaceae & $\mathrm{x}$ & $\mathrm{x}$ \\
\hline 413 & Smillax aspera $\mathrm{L}$. & Smilax aspera L. & Smilacaceae & $\mathrm{x}$ & $\mathrm{x}$ \\
\hline 414 & Solanum citrullifolium A. Bn. & Solanum citrullifolium A. Bn. & Solanaceae & & \\
\hline 415 & Solanum lycopersicum L. & Solanum lycopersicum L. & Solanaceae & $\mathrm{x}$ & $\mathrm{x}$ \\
\hline 416 & Solidago canadensis L. & Solidago canadensis L. & Asteraceae & $\mathrm{x}$ & \\
\hline 417 & Sonchus arvensis L. & Sonchus arvensis L. & Cichoriaceae & $\mathrm{x}$ & \\
\hline 418 & Sonchus asper (L.) Hill. & Sonchus asper (L.) Hill. & Cichoriaceae & $\mathrm{x}$ & $\mathrm{x}$ \\
\hline 419 & Sonchus oleraceus L. & Sonchus oleraceus L. & Cichoriaceae & $\mathrm{x}$ & \\
\hline 420 & Sophora Japonica L. & Sophora japonica L. & Fabaceae & $\mathrm{x}$ & \\
\hline 421 & Sorbus aria (L.) H. & Sorbus aria (L.) Crantz & Rosaceae & $x$ & $\mathrm{x}$ \\
\hline 422 & Sorghum halepense (L.) Pers. & Sorghum halepense (L.) Pers. & Poaceae & $\mathrm{x}$ & $x$ \\
\hline 423 & \begin{tabular}{|l|} 
Sorghum saccharatum (L.) \\
Pers., Sorghum vulgare L.
\end{tabular} & Sorghum bicolor (L.) Moench & Poaceae & $\mathrm{x}$ & \\
\hline 424 & Spartium junceum L. & Spartium junceum L. & Fabaceae & $x$ & $\mathrm{x}$ \\
\hline 425 & Spireae opulifolia L. & $\begin{array}{l}\text { Physocarpus opulifolius (L.) } \\
\text { Maxim. }\end{array}$ & Rosaceae & $x$ & \\
\hline 426 & Stachys annua L. & Stachys annua (L.) L. & Lamiaceae & $x$ & $\mathrm{x}$ \\
\hline 427 & Stachys arvensis L. & Stachys arvensis (L.) L. & Lamiaceae & $x$ & \\
\hline 428 & $\begin{array}{l}\text { Stachys officinalis (L.) } \\
\text { Trevisan }\end{array}$ & Stachys officinalis (L.) Trevis. & Lamiaceae & $\mathrm{x}$ & $\mathrm{x}$ \\
\hline 429 & Stachys salviaefolia Ten. & $\begin{array}{l}\text { Stachys cretica L. ssp. salviifolia } \\
\text { (Ten.) Rech. f. }\end{array}$ & Lamiaceae & $\mathrm{x}$ & $\mathrm{x}$ \\
\hline 430 & Stachys silvatica & Stachys sylvatica L. & Lamiaceae & $\mathrm{x}$ & $\mathrm{x}$ \\
\hline 431 & Staphylea pinnata & Staphylea pinnata L. & Staphyleaceae & $x$ & $\mathrm{x}$ \\
\hline 432 & Stellaria holostea L. & Stellaria holostea L. & Caryophyllaceae & $x$ & $\mathrm{x}$ \\
\hline 433 & Stellaria media $\mathrm{L}$. & Stellaria media (L.) Vill. & Caryophyllaceae & $x$ & $x$ \\
\hline 434 & Stipa pennata L. & Stipa pennata L. & Poaceae & $x$ & $\mathrm{x}$ \\
\hline 435 & Symphytum officinale & Symphytum officinale L. & Boraginaceae & $x$ & $x$ \\
\hline 436 & Syringa vulgaris L. & Syringa vulgaris L. & Oleaceae & $x$ & \\
\hline 437 & Tagetes erecta $\mathrm{L}$. & Tagetes erecta $\mathrm{L}$. & Asteraceae & $x$ & \\
\hline 438 & Tamus communis L. & Tamus communis L. & Dioscoreaceae & $\mathrm{x}$ & $\mathrm{x}$ \\
\hline 439 & Taraxacum Kok-saghyzi & Taraxacum kok-saghyz L.E.Rodin & Asteraceae & & \\
\hline 440 & Taraxacum officinale L. & Taraxacum officinale $\mathrm{L}$. & Cichoriaceae & $\mathrm{x}$ & \\
\hline 441 & Taxodium distichum $\mathrm{L}$. & Taxodium distichum (L.) Rich. & Taxodiaceae & $x$ & \\
\hline 442 & Taxus baccata $\mathrm{L}$. & Taxus baccata L. & Taxaceae & $\mathrm{x}$ & $\mathrm{x}$ \\
\hline 443 & Telekia speciosa Schreb. & $\begin{array}{l}\text { Telekia speciosa (Schreb.) } \\
\text { Baumg. }\end{array}$ & Asteraceae & $\mathrm{x}$ & $\mathrm{x}$ \\
\hline 444 & Thuja orientalis L. & Thuja orientalis L. & Cupressaceae & $x$ & \\
\hline
\end{tabular}




\begin{tabular}{|c|c|c|c|c|c|}
\hline No. & original name on label & valid name & family & HR & $\mathrm{ZAHO}$ \\
\hline 445 & Tilia argentea Desf. & Tilia tomentosa Moench & Tiliaceae & $x$ & \\
\hline 446 & Tordylium apulum $\mathrm{L}$. & Tordylium apulum $\mathrm{L}$. & Apiaceae & $\mathrm{x}$ & $\mathrm{x}$ \\
\hline 447 & Torilis arvensis (Huds.) Lk & Torilis arvensis (Huds.) Lk & Apiaceae & $x$ & \\
\hline 448 & Trachycarpus excelsa Wendl. & Rhapis excelsa (Thunb.) Henry & Arecaceae & & \\
\hline 449 & Tragopogon pratensis L. & Tragopogon pratensis L. & Cichoriaceae & $x$ & $x$ \\
\hline 450 & Trapa natans L. J. & Trapa natans L. & Trapaceae & $x$ & \\
\hline 451 & Trifolium arvense $\mathrm{L}$. & Trifolium arvense $\mathrm{L}$. & Fabaceae & $x$ & $x$ \\
\hline 452 & $\begin{array}{l}\text { Trifolium aureum, Trifolium } \\
\text { strepens Cr. }\end{array}$ & Trifolium aureum Pollich & Fabaceae & $x$ & $x$ \\
\hline 453 & Trifolium campestre Schre. & Trifolium campestre Schre. & Fabaceae & $x$ & $x$ \\
\hline 454 & Trifolium fragiferum $\mathrm{L}$. & Trifolium fragiferum $\mathrm{L}$. & Fabaceae & $x$ & \\
\hline 455 & Trifolium hybridum & Trifolium hybridum L. & Fabaceae & $x$ & $x$ \\
\hline 456 & Trifolium incarnatum & Trifolium incarnatum L. & Fabaceae & $x$ & $x$ \\
\hline 457 & Trifolium montanum $\mathrm{L}$. & Trifolium montanum L. & Fabaceae & $x$ & $x$ \\
\hline 458 & Trifolium pratense $\mathrm{L}$. & Trifolium pratense $\mathrm{L}$. & Fabaceae & $x$ & $x$ \\
\hline 459 & Trifolium rubens L. & Trifolium rubens L. & Fabaceae & $x$ & $x$ \\
\hline 460 & Trifolium stellatum L. & Trifolium stellatum $\mathrm{L}$. & Fabaceae & $\mathrm{x}$ & $\mathrm{x}$ \\
\hline 461 & Trifolium subterraneum L. & Trifolium subterraneum L. & Fabaceae & $x$ & \\
\hline 462 & Trollius europaeus L. & Trollius europaeus L. & Ranunculaceae & $\mathrm{x}$ & $x$ \\
\hline 463 & Tussilago farfara $\mathrm{L}$. & Tussilago farfara $\mathrm{L}$. & Asteraceae & $x$ & $x$ \\
\hline 464 & Typha latifolia L. & Typha latifolia L. & Typhaceae & $x$ & $x$ \\
\hline 465 & Onopordum acanthium L. & Onopordum acanthium L. & Asteraceae & $\mathrm{x}$ & \\
\hline 466 & Urtica dioica $\mathrm{L}$. & Urtica dioica $\mathrm{L}$. & Urticaceae & $x$ & $x$ \\
\hline 467 & Valeriana officinalis L. & Valeriana officinalis L. & Valerianaceae & $x$ & $x$ \\
\hline 468 & Veratrum album L. & Veratrum album L. & Melanthiaceae & $x$ & $x$ \\
\hline 469 & Verbascum floccosum W.K. & Verbascum pulverulentum Vill. & Scrophulariaceae & $x$ & \\
\hline 470 & Verbascum phlomoides L. & Verbascum phlomoides L. & Scrophulariaceae & $x$ & \\
\hline 471 & Verbena officinalis L. & Verbena officinalis L. & Verbanaceae & $x$ & $x$ \\
\hline 472 & Veronica officinalis L. & Veronica officinalis L. & Scrophulariaceae & $\mathrm{x}$ & $x$ \\
\hline 473 & Viburnum lantana $\mathrm{L}$. & Viburnum lantana $\mathrm{L}$. & Caprifoliaceae & $x$ & $x$ \\
\hline 474 & Viburnum opulus L. & Viburnum opulus L. & Caprifoliaceae & $x$ & $x$ \\
\hline 475 & Viburnum tinus L. & Viburnum tinus L. & Caprifoliaceae & $x$ & $x$ \\
\hline 476 & Vicia calcarata & Vicia monantha Retz. & Fabaceae & & \\
\hline 477 & Vicia ervilia (L.) Willd. & Vicia ervilia (L.) Willd. & Fabaceae & $\mathrm{x}$ & $x$ \\
\hline 478 & Vicia faba L. & Vicia faba L. & Fabaceae & $\mathrm{x}$ & \\
\hline 479 & Vicia lutea L. & Vicia lutea $\mathrm{L}$. & Fabaceae & $\mathrm{x}$ & $x$ \\
\hline 480 & \begin{tabular}{|l} 
Vicia sativa $\mathrm{L}$. \\
Vicia sativa ssp. obovata $\mathrm{H}$.
\end{tabular} & Vicia sativa $\mathrm{L}$. & Fabaceae & $x$ & $x$ \\
\hline 481 & Viola odorata L. & Viola odorata L. & Violaceae & $x$ & $x$ \\
\hline 482 & Vitex agnus-castus L. & Vitex agnus-castus L. & Verbanaceae & $\mathrm{x}$ & $\mathrm{x}$ \\
\hline 483 & Xanthium italicum Moretti. & $\begin{array}{l}\text { Xanthium orientale subsp. } \\
\text { italicum (Moretti) Greuter }\end{array}$ & Asteraceae & $x$ & \\
\hline 484 & Xanthium strumarium L. & Xanthium strumarium L. & Asteraceae & $x$ & $x$ \\
\hline 485 & Zea mays L. & Zea mays L. & Poaceae & $x$ & \\
\hline 486 & Ziziphus sativus Gaert. & Ziziphus jujuba Mill. & Rhamnaceae & $\mathrm{x}$ & \\
\hline
\end{tabular}

